\title{
ESTUDO COMPARATIVO DE MÉTODOS DE ESTIMAÇÃO DA VARIÂNCIA DE COEFICIENTE DE HERDABILIDADE
}

\author{
JOAQUIM PEREIRA NETO
}

Engenheiro Agrônomo

Orientador: PROF.DR. DECIO BARBIN

Dissertação apresentada à Escola Superior de Agricultura "Luiz de Queiroz", da Universidade de São Paulo, para a obtenção do título de Mestre em Agronomia, Área de Concentração: Estatística e Experimentação Agronômica.

P I R A C I C A B A

Estado de São Paulo - Brasil

Novembro - 1994 
Ficha cataloorafica preparada pela seça de Livros da

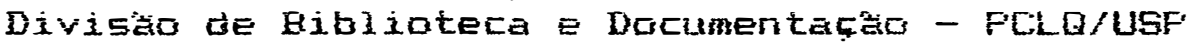

Fereira Weto, Joaquim

Fusbe Estudo comparativo de metodos de estimaçăo da variancia de coeficiente de herdabilidade. Fifacicata, 1994. 80p. ilus.

Diss. (MiEstre) - ESALQ

Bitiligrafia.

1. Anelise de variencia 2 . Estatistica aplicada 3. Herdatilidade I. Escula Superiof de fgricultura Luiz de turueiroz, Firaçicaba

CDD 519.535

575.1 


\section{ESTUDO COMPARATIVO DE MÉTODOS DE ESTIMAÇÃO DA VARIÂNCIA DE COEFICIENTE DE HERDABILIDADE}

JOAQUIM PEREIRA NETO

Aprovada em: 04.11 .94

Comissão julgadora:

Prof. Dr. Décio Barbin

ESALQ/USP

Profa. Dra. Clarice Garcia Borges Demétrio ESALQ/USP

Prof. Dr. Sérgio do Nascimento Kronka FACAV/UNESP

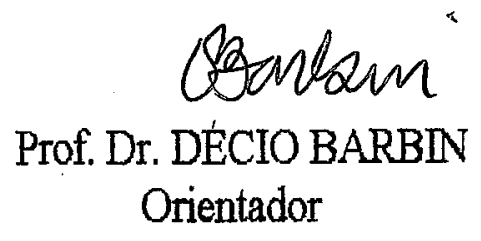


Este trabalho é dedicado

Aos meus pais,

Sancha e Antônio, pelo amor que me dedicaram.

A minha esposa,

Cida,

Às minhas filhas,

Jamille e Amanda, razão da minha dedicação. 


\section{AGRADECIMENTOS}

A Faculdade de Agronomia do Médio São Francisco, pela oportunidade.

À Escola Superior de Agricultura "Luiz de Queiroz", ESALQ, através do Departamento de Matemática e Estatistica.

À CAPES, através do PICD pela bolsa de estudo concedida.

Ao Prof. Titular do Departamento de Matemática e Estatistica da ESALQ, Dr. Décio Barbin pela orientação e sempre aberto ao dialógo.

A Profa. do Departamento de Matemática e Estatistica da ESALQ, Dra. Clarice Garcia Borges Demétrio, pela amizade e atenção dedicada.

Aos professores do Departamento de Matemática e Estatistica da ESALQ, pelos ensinamentos.

Ao amigo e colega da Faculdade de Agronomia do Médio São Francisco, Prof. Joston Simão de Assis pelo incentivo. 
Ao amigo do Curso de Doutorado, João Gil de Luna e sua esposa, sempre presentes a apoiar-me.

Aos amigos e colegas de Curso, Valdomiro, Marcos, Erivaldo, Ana, Marcia, Beatriz e Vera, pela amizade e assistência dedicadas.

Aos funcionários do Departamento de Matemática e Estatistica da ESALQ, pelo apoio prestado.

A0 amigo Antônio Raymundo Dantas Ramiro, diretor do Curtume Campelo S. A. em Juazeiro - BA, pelo apoio e incentivo.

Ao meu sogro Luiz Severiano da Costa, pela sua ajuda como meu procurador.

À Deus, que me deu forças para superar todos os momentos dificeis. 


\section{SUMÁRIO}

Página

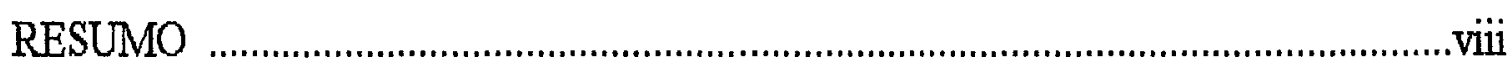

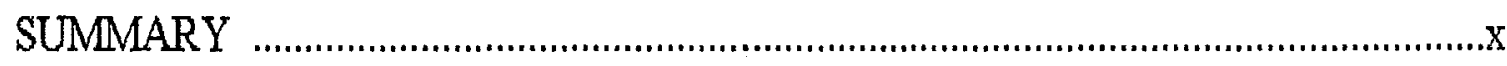

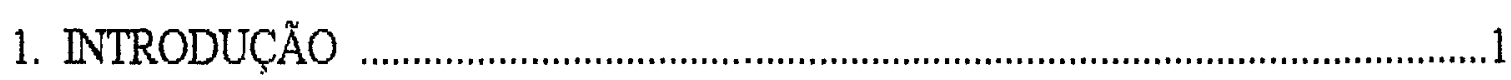

2. REVISÃO DE LITERATURA ......................................................................

2.1. Variância do coeficiente de herdabilidade ............................................................

2.2. Correlą̧ão genética intraclasse ……………………………………………......11

2.3. Estimativas negativas do coeficiente de herdabilidade ....................................15

2.4. Estimativas do coeficiente de herdabilidade maiores que $1\left(\mathrm{~h}^{2}>1\right) \ldots \ldots \ldots \ldots . . .18$

2.5. Intervalo de confiança para $\mathrm{H}^{2}$............................................................18

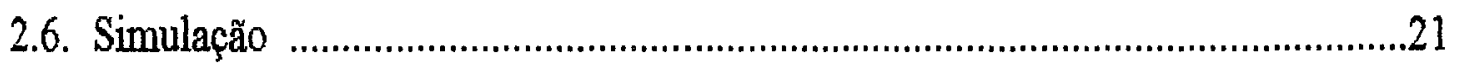

3. MATERIALE MÉTODOS _.............................................................................2

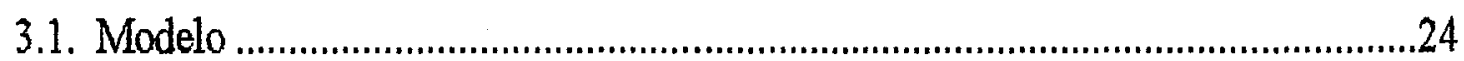

3.2. Análise de variância .....................................................................................25

3.3. Estimativa do coeficiente de herdabilidade .....................................................26

3.4. Variância do coeficiente de herdabilidade ……………………………………....26

3.5. Simulação dos dados de acordo com o modelo e experimentos.............................29

4. RESULTADOS E DISCUSSÃO ………………………………………….........32 


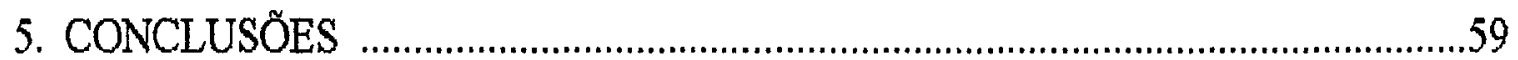

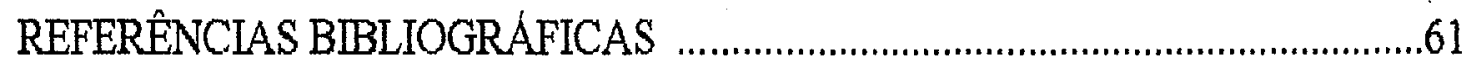

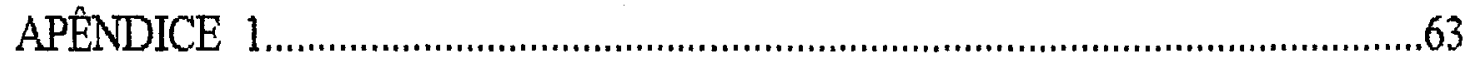

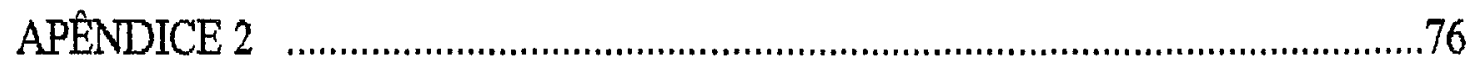




\title{
ESTUDO COMPARATIVO DE MÉTODOS DE ESTIMAÇÃO DA VARIÂNCIA DE COEFICIENTE DE HERDABILIDADE
}

\author{
Autor: JOAQUTM PEREIRA NETO \\ Orientador: PROF. DR DÉCIO BARBIN
}

\section{RESUMO}

O presente trabalho tem por objetivo fazer um estudo comparativo entre o emprego das formulas aproximadas de: OSBORNE \& PATERSON (1952), adaptada por BARBIN (1969), FALCONER (1960) e DICKERSON (1969), para o cálculo da variância do coeficiente de herdabilidade, empregando-se o modelo hierárquico aleatório.

Para encontrar as estimativas dos componentes de variância, aplicou-se o método dos momentos. As estimativas dos coeficientes de herdabilidade e das variâncias aproximadas, foram encontradas utilizando-se as estimativas dos componentes de variância e covariâncias.

Os dados foram obtidos através de simulação, adotando-se as metodologias de GRAYBILL et al. (1956), VANVLECK \& HENDERSON (1961) e GLL \& JENSEN (1968). Os desvios aleatórios foram obtidos com o emprego do gerador RANNOR do SAS (Statistical Analysis System). Foram simulados 50 experimentos para várias combinações de números de machos e fềmeas, com 2 
descendentes por acasalamento.

Dentre todas as situações simuladas, a fórmula de OSBORNE $\varepsilon$ PATERSON (1952), adaptada por BARBIN (1969), foi a que apresentou menor estimativa para a variância do coeficiente de herdabilidade. 


\title{
COMPARATIVE STUDY OF METHODS OF ESTIMATION OF HERITABILITY COEFFICIENT VARIANCE
}

\author{
Author: JOAQUIM PEREIRA NETO \\ Adviser: PROF. DR DÉCIO BARBIN
}

\section{SUMMARY}

The aim of this work is to make a comparative study between the approximated formulae of: OSBORNE \& PATERSON (1952), adapted by BARBN (1969), FALCONER (1960) and DICKERSON (1969) for the calculus of heritability coefficient variance by means of a random hierarchical model.

The momentum method was used in order to find the estimative of variance components. Approximate heritability coeficient and variance estimatives were found by estimating variance and covariance components.

Data were obtained through model simulation using the methodology of GRAYBILL et al. (1956), VANVLECK \& HENDERSON (1961), and GLL $\varepsilon$ JENSEN (1968), random deviations were obtained by a SAS (Statistical Analysis System) RANNOR generator . Fifty experiments were simulated for several combinations of sires and dams, with 2 descendants per mating. 
Among all simulated situations the formula by OSBORNE \& PATERSON (1952), adapted by BARBIN (1969), presented the lowest estimative for heritability coeficient variance. 


\section{INTRODUÇÃO}

Em Genética Estatistica estudam-se certas caracteristicas nos individuos, sem descontinuidades naturais, chamadas de "variação" ou "caracteres métricos", porque o estudo deles depende de mensuração, em vez de contagem. Qualquer atributo que varie continuamente, e suscetível de mensuração, poderia ser estudado, em principio, como uma caracteristica métrica, como: dimensões, proporções anatômicas, funções fisiológicas de toda a natureza e qualidades mentais ou psicológicas.

O estudo de um caráter métrico centraliza-se em torno da análise de sua variação, porque é em termos de variância que são formuladas as questões primárias da genética. $\mathrm{O}$ valor observado quando um caráter é medido no individuo, é o valor fenotipico desse individuo; todas as observaç̃es de médias, variâncias e covariâncias devem, claramente, ser baseadas na medida do valor fenotipico.

O coeficiente de herdabilidade de um caráter métrico é uma das mais importantes de suas propriedades. Ela expressa a proporção da variância total que é atribuida ao efeito médio dos gens, e este é o que determina o grau de semelhança entre os parentes. Mas, a mais importante função do coeficiente de herdabilidade no estudo genético do carater métrico è o seu papel preditivo, expressando a confiança do valor fenotipico como um guia para o valor genético. Somente o valor fenotipico do individuo 
pode ser diretamente medido, mas é o valor genético que determina a influência na próxima geração. Portanto, se o criador escolhe os individuos para serem pais, de acordo com os seus valores fenotípicos, seu sucesso na alteração das características da população pode ser predito somente por intermédio do conhecimento do grau de correspondência entre o valor do fenótipo e o valor genético. Este grau de correspondência é medido pelo coeficiente de herdabilidade, que será estimado pela técnica da análise da variância com o emprego do modelo hierárquico aleatório.

Devido a importância do coeficiente de herdabilidade nos programas de melhoramento animal, faz-se necessário um estudo mais detalhado das fórmulas disponiveis, todas aproximadas, para estimar a variância deste coeficiente. 


\section{REVISÃO DE LITERATURA}

\subsection{Variância do coeficiente de herdabilidade}

Os objetivos da estimação das variâncias genéticas são diversos. Um dos mais frequentes, particularmente entre os geneticistas da área animal, é a estimação do coeficiente de herdabilidade, um parâmetro definido como a razão da variância genética aditiva para a variância fenotipica, por isso ela expressa a proporção da variação total que é atribuida ao efeito médio dos gens. Mas, segundo BROEMELING (1969), o estudo da distribuição de estimadores do coeficiente de herdabilidade não tem sido examinado, devido ao desconhecimento da distribuição dos estimadores de componentes de variância.

OSBORNE \& PATERSON (1952), apresentam uma fórmula aproximada para a obtenção da variância do coeficiente de herdabilidade. Consideram o modelo de classificação hierárquica aleatório, conforme o quadro da análise da variância abaixo, com números constantes de observações por fềmea e por macho: 


\begin{tabular}{|c|c|c|c|}
\hline FONTE DE VARIAÇÃO & GL & QM & $\mathrm{E}[\mathrm{QM}]$ \\
\hline Entre machos & c & $\mathrm{M}_{3}$ & $\alpha+\mathrm{n} \beta+\gamma \mathrm{m}$ \\
\hline Entre fềmeas dentro de machos & $\mathrm{b}$ & $M_{2}$ & $\alpha+\mathrm{n} \beta$ \\
\hline Entre irmãos germanos & a & $\mathrm{M}_{1}$ & $\alpha$ \\
\hline
\end{tabular}

onde $\mathrm{n}$ e $\mathrm{m}$ são os números de individuos por fềmea e por macho, respectivamente. $\mathrm{A}$, $B$ e $C$ são as estimativas dos componentes de variância de $\alpha, \beta$ e $\gamma$ associados, respectivamente aos efeitos de individuos, fềmeas e machos. Considerando o quociente de duas variáveis aleatórias contínuas $\mathrm{X}$ e $\mathrm{Y}$ e, fazendo

$$
Z=\frac{X}{Y}
$$

usando a expansão em série de Taylor até os termos de primeira ordem, tem-se aproximadamente

$$
\delta_{z} \approx \frac{\bar{y} \delta_{x}-\bar{x} \delta_{y}}{\bar{y}^{2}},
$$

onde,

$\delta_{x}, \delta_{y}$ e $\delta_{z}$ são desvios da média.

Portanto,

$$
\sigma_{z}^{2} \frac{\left[\bar{y}^{2} \sigma_{x}^{2}+\bar{x}^{2} \sigma_{y}^{2}-2 \overline{x y} \operatorname{cov}(x, y)\right]}{\bar{y}^{4}} .
$$


Considerando-se que sob normalidade em modelos balanceados, qualquer quadrado médio $\mathrm{M}$ com $\mathrm{f}$ graus de liberdade é distribuido como

$$
\mathrm{QM} \sim \mathrm{E}[\mathrm{QM}] \frac{x_{\mathrm{f}}^{2}}{\mathrm{f}}
$$

e que os $\mathrm{QM}$ são mutuamente independentes, tem-se

$$
\begin{aligned}
& M_{1}=E\left[M_{1}\right] \frac{\chi_{a}^{2}}{a} \Rightarrow \sigma_{A}^{2}=\frac{2 A^{2}}{a}, \\
& M_{2}=\frac{1}{n}\left[E\left(M_{2}\right) \frac{\chi_{b}^{2}}{b}-E\left(M_{1}\right) \frac{\chi_{a}^{2}}{a}\right] \Rightarrow \sigma_{B}^{2}=\frac{2}{n^{2}}\left[\frac{(A+n B)^{2}}{b}+\frac{A^{2}}{a}\right],
\end{aligned}
$$

$e_{3}$

$$
\begin{aligned}
& M_{3}=\frac{1}{m}\left[E\left(M_{3}\right) \frac{\chi_{c}^{2}}{c}-E\left(M_{2}\right) \frac{\chi_{b}^{2}}{b}\right] \Rightarrow \\
& \sigma_{C}^{2}=\frac{2}{m^{2}}\left[\frac{(A+n B+C)^{2}}{c}+\frac{(A+n B)^{2}}{b}\right] .
\end{aligned}
$$

Seja a variável aleatória,

$$
\mathrm{H}^{2}=\frac{4 \mathrm{C}}{[\mathrm{A}+\mathrm{B}+\mathrm{C}]} \text {. }
$$


Substituindo $x$ e $y$ na fórmula (1) por $C$ e $(A+B+C)$, respectivamente, podemos encontrar a $\mathrm{V}\left(\mathrm{H}^{2}\right)$.

\section{Assim,}

$$
\begin{aligned}
& \sigma_{x}^{2}=\sigma_{C^{\prime}}^{2} \\
& \sigma_{y}^{2}=\sigma_{A}^{2}+\sigma_{B}^{2}+\sigma_{C}^{2}+2[\operatorname{cov}(A, B)+\operatorname{cov}(B, C)+\operatorname{cov}(A, C)], \\
& \operatorname{covv}(x, y)=\operatorname{covv}(A, C)+\operatorname{côv}(B, C)+\sigma_{C}^{2},
\end{aligned}
$$

cujas covariâncias são dadas por:

$$
\begin{aligned}
& \operatorname{cov}(A, A+n B)=\sigma_{A}^{2}+n \operatorname{covv}(A, B)=0 \quad \therefore \quad \operatorname{côv}(A, B)=-\frac{\sigma_{A}^{2}}{n}, \\
& \operatorname{covv}(A, A+n B+m C)=m \operatorname{covv}(A, C), \\
& \therefore \quad \operatorname{côv}(A, C)=0 \\
& \operatorname{covv}(A+n B, A+n B+m C)=\sigma_{A}^{2}+n^{2} \sigma_{B}^{2}+2 n \operatorname{covv}(A, B)+n m \operatorname{côv}(B, C)=0 \\
& \therefore \quad \operatorname{côv}(B, C)=\frac{\sigma_{A}^{2}-n^{2} \sigma_{B}^{2}}{n m} .
\end{aligned}
$$


FALCONER (1960), diz que se considerarmos um delineamento ideal, as variâncias de amostragem das estimativas do coeficiente de herdabilidade são as seguintes:

Para familias de irmãos completos: $\quad \sigma^{2}\left(\mathrm{~h}^{2}\right) \approx \frac{16 \mathrm{~h}^{2}}{\mathrm{~T}}$,

Para familias de meio-irmãos: $\quad \sigma^{2}\left(\mathrm{~h}^{2}\right) \approx \frac{32 \mathrm{~h}^{2}}{\mathrm{~T}}$,

onde,

$\mathrm{h}^{2}$, è a herdabilidade estimada pela análise da variância;

$T$, é o número total de observações.

BARBIN (1969), apresenta uma fórmula aproximada para o cálculo da estimativa da variância do coeficiente de herdabilidade, adaptada de OSBORNE $\varepsilon$ PATERSON (1952), para o caso de tamanhos desiguais de progênies. Esta adaptação constou de estabelecimento de fórmulas para o cálculo das variâncias e covariâncias dos componentes de variância. Assim,

$$
\begin{array}{r}
\hat{\mathrm{V}}\left(\mathrm{h}^{2}\right)=\frac{16}{(\mathrm{E}+\mathrm{D}+\mathrm{S})^{4}}\left[(\mathrm{E}+\mathrm{D})^{2} \hat{\mathrm{V}}(\mathrm{S})+\mathrm{S}^{2}[\hat{\mathrm{V}}(\mathrm{E})+\hat{\mathrm{V}}(\mathrm{D})+2 \operatorname{cov}(\mathrm{E}, \mathrm{D})]\right. \\
-2 \mathrm{~S}(\mathrm{E}+\mathrm{D})[\operatorname{cov}(\mathrm{E}, \mathrm{S})+\operatorname{cov}(\mathrm{D}, \mathrm{S})]] .
\end{array}
$$

Considerando,

$$
\hat{\mathrm{V}}_{1}=\mathrm{E}+\mathrm{k}_{2} \mathrm{D}+\mathrm{k}_{3} \mathrm{~S}
$$

com $\mathrm{n}_{1}=(\mathrm{I}-1)$ graus de liberdade, 


$$
\hat{\mathrm{V}}_{2}=\mathrm{E}+\mathrm{k}_{1} \mathrm{D}
$$

com $\mathrm{n}_{2}=(\mathrm{J}-1)$ graus de liberdade,

$$
\hat{\mathrm{V}}_{3}=\mathrm{E}
$$

com $\mathrm{n}_{3}=[\mathrm{N}-(\mathrm{I}+\mathrm{J}-1)]$ graus de liberdade, obteve

$$
\begin{aligned}
& \hat{V}(s)=\frac{2}{k_{1} k_{2}}\left[\frac{k_{1}^{2} \hat{V}_{1}^{2}}{n_{1}+2}+\frac{k_{2}^{2} V_{2}^{2}}{n_{2}+2}+\frac{\left(k_{2}-k_{1}\right)^{2}}{n_{3}+2}\right], \\
& \hat{V}(D)=\frac{2}{k_{1}^{2}}\left[\frac{\hat{V}_{2}^{2}}{n_{2}+2}+\frac{\hat{V}_{3}^{2}}{n_{3}+2}\right]
\end{aligned}
$$

e,

$$
\hat{V}(E)=\frac{2 \hat{V}_{3}^{2}}{n_{3}+2}
$$

Considerando a independência dos quadradros médios, obteve as estimativas das covariâncias:

$$
\begin{aligned}
& \operatorname{cov}\left(E, E+k_{1} D\right)=\hat{V}(E)+k_{1} \operatorname{cov}(E, D)=0, \\
& \therefore \quad \operatorname{cov}(E, D)=-\frac{\hat{V}(E)}{k_{1}} ;
\end{aligned}
$$




$$
\begin{aligned}
& \operatorname{cov}\left(E, E+k_{2} D+k_{3} S\right)=\hat{V}(E)+k_{2} \operatorname{cov}(E, D)+k_{3} \operatorname{cov}(E, S)=0, \\
& \therefore \quad \operatorname{cov}(E, S)=\frac{k_{2}-k_{1}}{k_{1} k_{3}} \hat{V}(E)
\end{aligned}
$$

e,

$$
\begin{aligned}
& \operatorname{côv}\left(E+k_{1} D, E+k_{2} D+k_{3} S\right)=\hat{V}(E)+k_{1} k_{2} \hat{V}(D)+\left(k_{1}+k_{2}\right) \operatorname{côv}(E, D) \\
&+k_{3} \operatorname{covv}(E, S)+k_{1} k_{3} \operatorname{côv}(D, S)=0, \\
& \therefore \quad \operatorname{cov}(D, S)=\frac{\hat{V}(E)-k_{1} k_{2} \hat{V}(D)}{k_{1} k_{3}} .
\end{aligned}
$$

DICKERSON (1969), sugeriu fórmulas aproximadas para a $\mathrm{V}\left(\mathrm{h}^{2}\right)$ com base na $v(x / y)$, desprezando os termos $v(y)$ e $\operatorname{cov}(x, y)$.

Assim,

$$
\begin{aligned}
& V\left(h_{S}^{2}\right)=\frac{16 V\left(\sigma_{S}^{2}\right)}{\left(\sigma_{S}^{2}+\sigma_{D}^{2}+\sigma_{W}^{2}\right)^{2}} \\
& V\left(h_{D}^{2}\right)=\frac{16 V\left(\sigma_{D}^{2}\right)}{\left(\sigma_{S}^{2}+\sigma_{D}^{2}+\sigma_{W}^{2}\right)^{2}}
\end{aligned}
$$

$\mathrm{e}_{\mathrm{g}}$

$$
V\left(h_{(S+D)}^{2}\right)=\frac{4\left[V\left(\sigma_{S}^{2}\right)+V\left(\sigma_{D}^{2}\right)+2 \operatorname{cov}\left(\sigma_{S}^{2}, \sigma_{D}^{2}\right)\right]}{\left(\sigma_{S}^{2}+\sigma_{D}^{2}+\sigma_{W}^{2}\right)^{2}} .
$$


SINGH (1993), considerando o modelo aleatório:

$$
\begin{aligned}
& y_{i j}=m+a_{i}+e_{i j}, \\
& \left(j=1,2, \ldots, n_{i} ; i=1,2, \ldots, g, \sum_{i=1}^{g} n_{i}=N\right),
\end{aligned}
$$

onde,

$$
\begin{aligned}
& \mathrm{m}=\text { média geral, } \\
& \mathrm{a}_{\mathrm{i}}=\text { efeito do grupo } \mathrm{i}, \mathrm{a}_{\mathrm{ij}} \sim \operatorname{NIID}\left(0, \sigma_{\mathrm{a}}^{2}\right), \\
& \mathrm{e}_{\mathrm{ij}}=\text { efeito residual, } \mathrm{e}_{\mathrm{ij}} \sim \mathrm{NIID}\left(0, \sigma_{\mathrm{e}}^{2}\right),
\end{aligned}
$$

encontra a distribuição exata para a variável aleatória,

$$
H^{2}=\frac{4 \sigma_{a}^{2}}{\left[\sigma_{a}^{2}+\sigma_{e}^{2}\right]} .
$$

Ainda mais, encontra os momentos da função de densidade de $f\left(h^{2}\right)$ e, faz um estudo comparativo entre a variância exata de $h^{2}$ e a variância estimada pelas fórmulas aproximadas de Taylor, Smith e Fisher, considerando os valores esperados para a herdabilidade de 0,$0 ; 0,2 ; 0,4 ; 0,6 ; 0,8$ e 1,0 . Conclui que a fórmula de Smith para a $\mathrm{V}\left(\mathrm{h}^{2}\right)$ é a que apresentou melhor precisão para todas as situações de tamanhos de grupos. 
KEMPTHORNE (1957), considerando o modelo de classificação hierárquica balanceado aleatório,

$$
y_{i j k}=\mu+b_{i}+f_{i j}+s_{i j k}+e_{i j k}
$$

onde,

$$
\mathrm{i}=1,2, \ldots, \mathrm{b} ; \quad \mathrm{j}=1,2, \ldots, \mathrm{f} ; \quad \mathrm{k}=1,2, \ldots, \mathrm{s} ;
$$

\begin{tabular}{|c|c|c|c|}
\hline FONTE DE VARIAÇÃO & GL & QM & $\mathrm{E}[\mathrm{QM}]$ \\
\hline Raças & $b-1$ & M.S.B & $\sigma_{e}^{2}+r \sigma_{s}^{2}+\pi s \sigma_{f}^{2}+r s f \sigma_{b}^{2}$ \\
\hline Granjas dentro de raças & $b(f-1)$ & M.S.F & $\sigma_{\mathrm{e}}^{2}+r \sigma_{\mathrm{s}}^{2}+r s \sigma_{\mathrm{f}}^{2}$ \\
\hline Machos dentro de granjas & $b f(s-1)$ & M.S.S & $\sigma_{\mathrm{e}}^{2}+r \sigma_{s}^{2}$ \\
\hline Individuos dentro de machos & $b f(t-1)$ & M.S.I & $\sigma_{\mathrm{e}}^{2}$ \\
\hline TOTAL & bfsr-1 & & \\
\hline
\end{tabular}

e a tabela da análise da variância abaixo:

empregando a técnica da análise de variância, mostra como encontrar a estimativa da correlação intraclasse. Uma estimativa da correlação intraclasse é dada por:

$$
\hat{\theta}=\frac{n_{1} A+n_{2} B+n_{3} C+\ldots}{v_{1} A+v_{2} B+v_{3} C+\ldots}=\frac{X}{Y}
$$

onde, 


$$
\begin{aligned}
& \mathrm{n}_{1}, \mathrm{n}_{2}, \mathrm{n}_{3}, \ldots, \\
& \mathrm{v}_{1}, \mathrm{v}_{2}, \mathrm{v}_{3}, \ldots, \text { são números reais }
\end{aligned}
$$

e

$$
A, B \text { e } C \text {, são quadrados médios na análise de variância. }
$$

Com base no modelo e na tabela da análise da variância, define o coeficiente de correlação intraclasse,

$$
\rho=\frac{\sigma_{f}^{2}}{\sigma_{e}^{2}+\sigma_{s}^{2}+\sigma_{f}^{2}}
$$

e sua estimativa por

$$
\hat{\rho}=\frac{1 / \mathrm{rs}(\text { M.S.F }- \text { M.S.S })}{\text { M.S.I+(M.S.S-M.S.I)/r+(M.S.F-M.S.S }) / \mathrm{rs}}
$$

Em seguida mostra a relação aproximada,

$$
\mathrm{V}(\hat{\theta})=\frac{\mathrm{V}(\mathrm{X})}{\mathrm{Y}^{2}}-2 \frac{\mathrm{X} \operatorname{cov}(\mathrm{X}, \mathrm{Y})}{\mathrm{Y}^{3}}+\frac{\mathrm{X}^{2}}{\mathrm{Y}^{4}} \mathrm{~V}(\mathrm{Y})
$$

onde,

$$
\begin{aligned}
& V(X)=u_{1}^{2} V(A)+u_{2}^{2} V(B)+u_{3}^{2} V(C)+\cdots, \\
& V(Y)=v_{1}^{2} V(A)+v_{2}^{2} V(B)+v_{3}^{2} V(C)+\cdots \quad e,
\end{aligned}
$$


ROBERTSON (1959), considerando um delineamento com uma única classificação, com $\mathrm{N}$ familias e com n meio-irmãos por familia, fez uso da fórmula de FISHER (1954) para encontrar a variância da correlação intraclasse t ,

$$
v(\hat{t}) \approx \frac{2[1+(n-1) t]^{2}(t-1)^{2}}{n(n-1)(N-1)}
$$

Impondo a restrição $\mathrm{Nn} \leq \mathrm{T}$ e fazendo-se $\mathrm{n}$ grande, tem-se,

$$
\mathrm{V}(\hat{\mathrm{t}}) \approx \frac{2(1-\mathrm{t})^{2}(1+\mathrm{nt})^{2}}{\mathrm{Tn}}
$$

Considerando,

$$
V(\hat{t})=f(n)
$$

tem-se que $v(\hat{t})$, admite um minimo quando $n t=1$. Portanto, 0 autor chegou à conclusão que o tamanho ótimo do grupo ocorre quando $n=1 / t$. Admitindo o ótimo, tem-se

$$
\begin{aligned}
V(\hat{\mathbf{t}}) & \approx \frac{8(1-t)^{2}}{T} \\
& \approx \frac{8 \mathrm{t}^{2}}{\mathrm{~N}} \\
& \approx \frac{8 \mathrm{t}}{\mathrm{T}} .
\end{aligned}
$$


No caso do modelo de classificação hierárquica com $\mathrm{s}$ machos, $\mathrm{d}$ fềmeas por macho $\mathrm{e}$ $\mathrm{n}$ descendentes por fềmea, respectivamente, seguindo o trabalho de OSBORNE $\varepsilon$ PATERSON (1952), concluiu que:

a) a melhor estimativa da correlação intraclasse para machos ocorre quando se tem uma progênie por fềmea $\mathrm{e} o$ número de descendentes por macho é aproximadamente $1 / \mathrm{t}$.

b) para uma btima e igual informação de ambas as correlações, 0 delineamento deverá ter de 3 a 4 fềmeas por macho e $1 / 2 t$ descendentes por fềmea. $\mathrm{Na}$ discussão do seu trabalho, diz que, com uma ótima estrutura para a análise estatistica, a variância do coeficiente de herdabilidade é dada por:

$$
\hat{\mathrm{V}}(\hat{\mathrm{t}}) \approx \frac{8 \mathrm{t}}{\mathrm{T}} \quad \text { ou } \quad \hat{\mathrm{V}}\left(\mathrm{h}^{2}\right) \approx \frac{32 \mathrm{~h}^{2}}{\mathrm{~T}}
$$

onde $\mathrm{T}$ é o número total de observações.

SWINGER et al. (1964), trabalhando com o coeficiente de correlação genética intraclasse, considerando números de observações diferentes por grupo, com base no quadro da análise da variância abaixo:

\begin{tabular}{|c|c|c|c|}
\hline FONTE DE VARIAÇÃO & GL & SQ & $\mathrm{E}[\mathrm{QM}]$ \\
\hline Entre grupos & $s-1$ & $s$ & $(s-1)\left(\sigma_{e}^{2}+k \sigma_{g}^{2}\right)$ \\
\hline Dentro dos grupos & $N-s$ & E & $(\mathrm{N}-\mathrm{s}) \sigma_{\mathrm{e}}^{2}$ \\
\hline
\end{tabular}


e na fórmula aproximada para o quociente entre duas variáveis aleatórias $\mathrm{X}$ e $\mathrm{Y}$,

$$
V\left(\frac{Y}{X}\right) \approx\left(\frac{\mu_{y}}{\mu_{x}}\right)^{2}\left(\frac{\sigma_{y}^{2}}{\mu_{y}^{2}}+\frac{\sigma_{x}^{2}}{\mu_{x}^{2}}-2 \frac{\operatorname{cov}(x, y)}{\mu_{x} \mu_{y}}\right)
$$

chegaram à seguinte expressão aproximada, para a variância do coeficiente de correlação genética intraclasse:

$$
V(\hat{t}) \approx \frac{2(N-1)(1-t)^{2}[1+(k-1) t]^{2}}{k^{2}(N-s)(s-1)}
$$

onde,

$$
t=\frac{\hat{\sigma}_{g}^{2}}{\hat{\sigma}_{g}^{2}+\hat{\sigma}_{e}^{2}} \quad \text { e } \quad k=\frac{1}{s-1}\left(N-\frac{\sum n_{i}^{2}}{N}\right)
$$

Assumindo número igual de observações por grupo, a expressão reduz-se à fórmula de FISHER (1954).

\subsection{Estimativas negativas do coeficiente de herdabilidade}

Um componente de variância é, por definição, sempre positivo. Entretanto, não é incomun a ocorrência de componentes de variância negativos, levando a estimativas também negativas do coeficiente de herdabilidade, o que acontece quando a variância residual é maior que a variância entre grupos paternos ou maternos, quer em 
modelos balanceados ou não balanceados.

NELDER (1954), diz que, se o modelo na análise é o modelo normal ortodoxo, a prática de igualar a zero os componentes de variâncias negativos, é muito menos questionável e não conduz a vieses maiores na estimação dos erros padrões, etc. Destaca ainda, que num experimento envolvendo um número pequeno de observações, podem ocorrer problemas de correlações, conduzindo a tais estimativas negativas.

KEMPTHORNE (1968), comenta: "não existe qualquer objeção lógica ao emprego de uma estatistica que possa assumir valores negativos, numa tentativa de obter informações especificas a respeito de um parâmetro não negativo". E, continua "se diversos conjuntos de dados fornecem uma mistura de estatisticas positivas e negativas, pode-se presumir que o verdadeiro valor comum está em torno de zero."

GLL $\varepsilon$ JENSEN (1968), trabalhando com estimativas negativas do coeficiente de herdabilidade, chegaram às seguintes conclusões:

i) Para um determinado valor do coeficiente de herdabilidade e números iguais do total de observações, a probabilidade de se obter uma estimativa negativa do componente fềmea (dentro de macho) em uma análise com dois irmãos germanos por acasalamento, é muito maior do que a do componente macho em qualquer análise com irmãos completos ou meios-irmãos.

ii) Quando um coeficiente de herdabilidade é estimado de um componente macho, a diferença em probabilidade, de se obter uma estimativa negativa da análise de meio-irmãos ou irmãos-completos é menor, se são iguais os totais de observações, mas existe alguma vantagem em usar mais informação por macho, 
especialmente se o coeficiente de herdabilidade é baixo.

iii) Se o coeficiente de herdabilidade é relativamente baixo, em torno de $(0,1)$, necessitamos no minimo de 800 observações para ter $95 \%$ de chance de se obter uma estimativa não-negativa do componente macho. Se a informação por macho é limitada a menos que 30 - 40 progênies, HILL (1965), mostra que uma estimativa não viesada, negativa, grande de um componente de variância é, provavelmente, uma indicação de que o experimento é não informativo, e não que o componente de variância seja aproximadamente zero.

iv) Se o coeficiente de herdabilidade é moderado, em torno de $(0,25)$, necessita-se de, no minimo, 500 observações para ter $99 \%$ de chance de se obter uma estimativa não-ṇegativa do componente macho; se a informação por macho é limitada a menos que $30-40$ progênies.

v) Aproximadamente, quatro vezes mais observações são necessárias para estimar componentes por fềmea (dentro de machos e com dois irmãos completos por acasalamento), do que componentes por macho, para alcançar a mesma probabilidade de se obter uma estimativa não negativa do coeficiente de herdabilidade. (Nota: A melhor maneira de melhorar estimativas para o componente fềmea será pelo aumento do número de irmãos completos por acasalamento, do que pelo aumento do número de fêmeas).

Conclui que existe alguma vantagem em planejar mais descendentes por macho do que avaliar mais machos (para um dado numero de descendentes). 


\subsection{Estimativas do coeficiente de herdabilidade maiores que $1\left(h^{2}>1\right)$.}

Segundo SLVA (1982), este é um problema que eventualmente ocorre, sendo aparentemente devido a um elevado valor da variância entre progênies, em relação à variância residual, o que pode ser causado por:

a) um número de graus de liberdade excessivamente pequeno entre progênies;

b) uso de amostras populacionais muito pequenas;

c) presença de fatores não aditivos ou não genéticos, contribuindo para uma variância entre progênies excessivamente inflacionada.

Ainda, segundo SILVA (1982), de um modo geral, trata-se de um resultado que indica inadequação da amostra para fins de estimação de parâmetros genéticos, os quais, nesse caso, não terão qualquer significado.

Devido ao desconhecimento da distribuição de probabilidade da variável aleatória $\mathrm{H}^{2}$ e, consequentemente, do seu dominio, reveste-se de importância admitir-se a ocorrência de coeficientes de herdabilidade maiores que 1.

\subsection{Intervalo de confiança para $\mathrm{H}^{2}$}

GRAYBLL et al. (1956), considerando o modelo de classificação hierárquica aleatório 


$$
y_{i j k}=\mu+a_{i}+b_{i j}+c_{i j k}
$$

onde,

$$
\mathrm{i}=1,2, \ldots, \mathrm{I} ; \quad \mathrm{j}=1,2, \ldots, \mathrm{s} \quad \text { e } \quad \mathrm{k}=1,2, \ldots, \mathrm{t}
$$

\begin{tabular}{|c|c|c|c|}
\hline FONTE DE VARIAÇÃO & GL & $Q M$ & $E[Q M]$ \\
\hline Entre machos & $\mathrm{n}_{3}=\mathrm{r}-1$ & $A_{3}$ & $\sigma_{3}^{2}=\sigma_{c}^{2}+t \sigma_{b}^{2}+s t \sigma_{a}^{2}$ \\
\hline Entre fềmeas dentro de machos & $\mathrm{n}_{2}=\mathrm{r}(\mathrm{s}-1)$ & $A_{2}$ & $\sigma_{2}^{2}=\sigma_{c}^{2}+t \sigma_{b}^{2}$ \\
\hline Entre irmãos germanos & $\mathbf{n}_{1}=\mathbf{r s}(\mathrm{t}-1)$ & $A_{1}$ & $\sigma_{1}^{2}=\sigma_{c}^{2}$ \\
\hline
\end{tabular}

e a tabela da análise da variância abaixo:

apresentam um método para encontrar intervalos de confiança para a variável aleatória,

$$
\mathrm{H}^{2}=\frac{2\left(\sigma_{\mathrm{a}}^{2}+\sigma_{b}^{2}\right)}{\left[\sigma_{\mathrm{a}}^{2}+\sigma_{b}^{2}+\sigma_{c}^{2}\right]}
$$

O método consiste em igualar os momentos de

$$
\frac{Y}{N}=\frac{\alpha_{2} A_{2}+\alpha_{3} A_{3}}{\sigma_{4}^{2}}
$$

aos momentos da função $\chi(\mathrm{N}) / \mathrm{N}, \quad$ onde 
$\sigma_{4}^{2}=\gamma\left(\sigma_{a}^{2}+\sigma_{b}^{2}\right)+\sigma_{c}^{2}$

$\mathrm{Y} / \mathrm{N}$ é uma combinação linear de variáveis com distribuição $\chi^{2}$ que é aproximada por $\chi_{(\mathrm{N})}^{2} / \mathrm{N}$,

$N_{3} \alpha_{2}, \alpha_{3}$ e $\gamma$ são constantes a determinar.

Considerando as funções geradoras,

$$
M(Y / N)(\theta)=\left(1-2 B_{2} \theta\right)^{-n_{2} / 2}\left(1-B_{3} \theta\right)^{-n_{3} / 2}
$$

e,

$$
M\left(\chi^{2} / \mathrm{N}\right)^{(\theta)=(1-2 \theta / N)^{-N / 2}}
$$

onde

$$
\mathrm{B}_{\mathrm{i}}=\frac{\alpha_{\mathrm{i}} \sigma_{\mathrm{i}}^{2}}{\mathrm{n}_{\mathrm{i}} \sigma_{4}^{2}}
$$

expandindo em séries infinitas e igualando aos primeiros momentos dessas funções temse,

$$
\gamma=\mathrm{t}, \quad \alpha_{2}=(\mathrm{s}-1) \quad \text { e } \quad \alpha_{3}=1 / \mathrm{s}
$$

Para obter um intervalo de confiança aproximado para $\mathrm{H}^{2}$, segue-se que

$$
\mathrm{Y} \sim \chi_{(\mathrm{N})}^{2} \quad \text { e } \quad \mathrm{n}_{1} \mathrm{~A}_{1} / \sigma_{\mathrm{c}}^{2} \sim \chi_{\left(\mathrm{n}_{1}\right)}^{2}
$$

Portanto,

$$
\frac{Y}{N} / \frac{A_{1}}{\sigma_{c}^{2}}=u
$$


tem distribuição aproximada de $F$ com $N$ graus de liberdade no numerador e $n_{1}$ graus de liberdade no denominador.

$$
\begin{aligned}
& \text { Sejam, } F_{1} \text { e } F_{2} \text { valores tais que } \\
& \int_{0}^{F_{1}} f(u) d u=\int_{F_{2}}^{\infty} f(u) d u=\alpha,
\end{aligned}
$$

usando esta igualdade, tem-se

$$
\mathrm{P}\left[\mathrm{F}_{1} \leq \mathrm{u} \leq \mathrm{F}_{2}\right]=1-2 \alpha .
$$

Substituindo u e fazendo algumas operações algébricas, obtém-se

$$
\mathrm{P}\left[\frac{2\left(\mathrm{C}-\mathrm{F}_{2}\right)}{\mathrm{C}+(\mathrm{t}-1) \mathrm{F}_{2}} \leq \mathrm{h}^{2} \leq \frac{2\left(\mathrm{C}-\mathrm{F}_{1}\right)}{\mathrm{C}+(\mathrm{t}-1) \mathrm{F}_{1}}\right]=(1-2 \alpha)
$$

onde,

$$
\mathrm{C}=\frac{(\mathrm{s}-1) \mathrm{A}_{2}+\mathrm{A}_{3}}{\mathrm{sA_{1 }}}
$$

\subsection{Simulaçã̃o}

GRAYBLL et al. (1956), considerando o modelo de classificação aleatório e vários casos onde fazem combinações do número de machos, fềmeas $\mathrm{e}$ descendentes, empregam a técnica da simulação para a obtenção dos dados. 
SWNGER et al. (1964), estudando a variância da correlação intraclasse, empregam a técnica de Monte Carlo para a simulação das observaç̃es.

GLL \& JENSEN (1968), estudando estimativas negativas do coeficiente de herdabilidade, considerando o modelo de classificação hierárquica aleatório, empregam a técnica da simulação e sugerem o trabalho de VANVLECK $\varepsilon$ HENDERSON (1961).

VANVLECK \& HENDERSON (1961), consideram o modelo

$$
y_{i j}=\mu+\alpha_{i}+\delta_{i j}, \quad\left(i=1,2, \ldots c ; j=1,2, \ldots n_{i}\right)
$$

onde,

$\mu$ é uma constante,

$\alpha_{i}$ é o efeito aleatório associado a $i$ - ésima classe ,

$\delta_{i j}$ é o efeito aleatório associado a j-ésima observação na i-ésima

classe,

e

$$
\begin{aligned}
& \alpha_{\mathrm{i}} \sim N\left(0, \sigma_{\alpha}^{2}\right), \\
& \delta_{\mathrm{ij}} \sim N\left(0, \sigma_{\delta}^{2}\right),
\end{aligned}
$$

Afirmam que, para gerar observações associadas a este modelo, deve-se retirar amostras de uma população $\mathrm{ND}(0,1)$, multiplicar estes desvios normais por um apropriado desvio padrão e adicionar estes produtos à média populacional. 
Assim,

$$
y_{i j}=\mu+\sigma_{\alpha} a_{i}+\sigma_{\delta} e_{i j}
$$

onde,

$$
a_{i} \sim \operatorname{NID}(0,1),
$$

$\mathrm{e}_{\mathrm{ij}} \sim \mathrm{NID}(0,1)$. 


\section{MATERIAL E MÉTODOS}

\subsection{Modelo}

Uma forma comum, por meio da qual os dados (características métricas), são obtidos com animais, é a seguinte: vários machos (reprodutores) são acasalados, cada um com diversas fềmeas (reprodutoras) diferentes e os descendentes de cada fềmea são medidos, fornecendo os dados experimentais. Portanto, para estimar a variância associada a estes efeitos $\mathrm{e}$, consequentemente o coeficiente de herdabilidade, foi empregado o modelo de classificação hierárquica aleatório, expresso por:

$$
\mathrm{y}_{\mathrm{ijk}}=\mu+\alpha_{\mathrm{i}}+\beta_{\mathrm{ij}}+\mathrm{e}_{\mathrm{ijk}}
$$

onde $\mu$ é fixo e representa a média geral e

$\alpha_{i}$, com $i=1,2, \ldots, I$ representa o efeito de machos com média zero e variância $\sigma_{\alpha}^{2}$;

$\beta_{\mathrm{ij}}$, com $\mathrm{j}=1,2, \ldots, \mathrm{J}$ representa o efeito de fềmeas dentro de machos com média zero e variância $\sigma_{\beta}^{2}$; 
$\mathrm{e}_{\mathrm{ijk}}$, com $\mathrm{k}=1,2, \ldots, \mathrm{K}$ representa o efeito de irmãos germanos, com média zero e variância $\sigma^{2}$.

\subsection{Análise de variância}

Considerando o modelo empregado temos o seguinte esquema de análise da variância:

\begin{tabular}{|c|c|c|c|}
\hline FONTE DE VARIAÇÃO & GL & $Q M$ & $\mathrm{E}[\mathrm{QM}]$ \\
\hline Entre machos & $I-1$ & $A_{3}$ & $\sigma^{2}+\mathrm{K} \sigma_{\beta}^{2}+\mathrm{JK} \sigma_{\alpha}^{2}$ \\
\hline Entre fêmeas dentro de machos & $(\mathrm{J}-1) \mathrm{I}$ & $A_{2}$ & $\sigma^{2}+K \sigma_{\beta}^{2}$ \\
\hline Entre irmấos germanos & $(\mathrm{K}-1) \mathrm{IJ}$ & $A_{1}$ & $\sigma^{2}$ \\
\hline TOTAL & $N-1$ & & \\
\hline
\end{tabular}

Para encontrar as estimativas dos componentes de variância, foi empregado o método dos momentos. Para facilitar o programa computacional, admitiu-se que

$$
\begin{aligned}
& E=\text { estimativa de } \sigma^{2}, \\
& D=\text { estimativa de } \sigma_{\beta}^{2}, \\
& S=\text { estimativa de } \sigma_{\alpha}^{2} .
\end{aligned}
$$

Logo, as estimativas dos componentes foram expressas por: 


$$
\begin{aligned}
& E=A_{1}, \\
& D=\frac{1}{K}\left(A_{2}-A_{1}\right)
\end{aligned}
$$

e

$$
S=\frac{1}{\sqrt{K}}\left(A_{3}-A_{2}\right)
$$

\subsection{Estimativa do coeficiente de herdabilidade}

Existe um número relativamente grande de métodos propostos para 0 cálculo do coeficiente de herdabilidade. O método empregado foi o da correlação entre meios-irmãos paternos que constitui, provavelmente, o método mais usado, tendo em vista possibilitar o aproveitamento de maior quantidade de dados. De fato, entre os animais domésticos é geralmente mais fácil encontrar um grande número de grupos de meios irmãos-completos do que irmãos completos. $O$ coeficiente de herdabilidade, no sentido restrito, é então estimado por:

$$
h^{2}=\frac{4 S}{E+D+S}
$$

\subsection{Variância do coeficiente de herdabilidade}

Geralmente, a variância amostral de estatisticas baseadas em componentes de variância é elevada e desconhecida; em alguns casos, a determinação 
dessa variância, com precisão suficientemente grande, chega a ser impossivel. Desta maneira, devido ao desconhecimento da distribuição de probabilidades da variavel aleatória $\mathrm{H}^{2}$, as fómulas existentes com este propósito são quase todas aproximadas. Como o objetivo é fazer um estudo comparativo de fórmulas aproximadas, foram empregadas as seguintes formulas para o cálculo da variância de $h^{2}$, baseadas na correlação entre meios-irmãos paternos:

a) OSBORNE $\&$ PATERSON (1952) adaptada por BARBIN (1969):

onde,

$$
\begin{array}{r}
\hat{V}\left(h^{2}\right) \approx \frac{16}{(E+D+S)^{4}}\left[(E+D)^{2} \hat{V}(S)+S^{2}[\hat{V}(E)+\hat{V}(D)+2 \operatorname{covv}(E, D)]\right. \\
-2 S(E+D)[\operatorname{cov}(E, S)+\operatorname{cov}(D, S)]]
\end{array}
$$

$$
\begin{aligned}
& \hat{\mathrm{V}}(\mathrm{E})=\frac{2 \mathrm{~V}_{3}^{2}}{(\mathrm{~K}-1) \mathrm{IJ}+2}, \\
& \hat{\mathrm{V}}(\mathrm{D})=\frac{2}{\mathrm{~K}^{2}}\left[\frac{(\mathrm{E}+\mathrm{KD})^{2}}{(\mathrm{~J}-1) \mathrm{I}+2}+\frac{\mathrm{E}^{2}}{(\mathrm{~K}-1) \mathrm{IJ}+2}\right], \\
& \hat{\mathrm{V}}(\mathrm{S})=\frac{2}{(\mathrm{JK})^{2}}\left[\frac{(\mathrm{E}+\mathrm{KD}+\mathrm{KKS})^{2}}{(\mathrm{I}-1)+2}+\frac{(\mathrm{E}+\mathrm{KD})^{2}}{(\mathrm{~J}-1) \mathrm{I}+2}\right]
\end{aligned}
$$

e,

$$
\operatorname{côv}(E, D)=-\frac{2}{K}\left[\frac{V_{3}}{(K-1) I J+2}\right] \text {, }
$$




$$
\operatorname{côv}(E, S)=0,
$$

$\operatorname{cov}(D, S)=-\frac{2}{J^{2}}\left[\frac{V_{2}^{2}}{(J-1) I+2}\right]$

b) FALCONER (1960):

$$
\hat{V}\left(h^{2}\right) \propto \frac{32 h^{2}}{T}
$$

onde,

$\mathrm{h}^{2}$, é o coeficiente de herdabilidade estimado,

$\mathrm{T}$, é o número total de observações.

c) DICKERSON (1969):

$$
\hat{V}\left(h^{2}\right) \approx \frac{16 \hat{V}(s)}{(E+D+s)^{2}}
$$

onde,

$\mathrm{E}, \mathrm{D}$, e S são estimativas dos componentes de variância para irmãos germanos, fềmeas dentro de machos e machos, respectiva mente.

$\hat{\mathrm{V}}(\mathrm{S}), \quad$ é a estimativa da variância de $\mathrm{S}$. 


\subsection{Simulação dos dados de acordo com o modelo e experimentos}

Para simulação dos dados, considerou-se as metodologias empregadas por GRAYBLL et al. (1956), VANVLECK \& HENDERSON (1961) e GILL \& JENSEN (1968). Assim, considerando o modelo aleatório hierárquico:

$$
y_{i j k}=\mu+\alpha_{i}+\beta_{i j}+e_{i j k}, i=1,2, \ldots, I ; j=1,2, \ldots, J ; k=1,2, \ldots, K
$$

onde,

$\mu_{2}$ é uma constante;

$\alpha_{i}, \quad$ é o efeito do $\mathrm{i}$ - ésimo macho; $\alpha_{\mathrm{i}} \sim \mathrm{NID}\left(0, \sigma_{\alpha}^{2}\right)$;

$\beta_{\mathrm{ij}}$, é o efeito da $\mathrm{j}$ - ésima fềmea dentro do $\mathrm{i}$ - ésimo macho;

$$
\beta_{\mathrm{ij}} \sim \mathrm{NID}\left(0, \sigma_{\beta}^{2}\right) \text {; }
$$

$e_{\mathrm{ijk}}$, é o efeito residual; $\mathrm{e}_{\mathrm{ijk}} \sim \mathrm{NID}\left(0, \sigma^{2}\right)$.

A variância de $y_{i j k}$ é $\left(\sigma_{\alpha}^{2}+\sigma_{\beta}^{2}+\sigma^{2}\right)$. Assim, para gerar observações associadas a este modelo, retirárou-se amostras de uma população $\mathrm{ND}(0,1)$, multiplicou-se estes desvios normais aleatórios por um apropriado desvio padrão e, adicionou-se estes produtos à média populacional.

Logo, o modelo pôde ser escrito como:

$$
y_{i j k}^{\prime}=\mu+\sigma_{\alpha} a_{i}+\sigma_{\beta} b_{i j}+\sigma e_{i j k}
$$

onde,

$$
a_{i}, b_{i j} \text { e } e_{i j k} \sim N D(0,1) \text {. }
$$


Foi considerado 0 valor de $\mu=15$, com o objetivo de eliminar valores negativos para os dados simulados e, os valores de $\sigma_{\alpha}, \sigma_{\beta}$ e $\sigma$, foram escolhidos de tal forma a dar valores esperados para os coeficientes de herdabilidade de 0,$0 ; 0,2 ; 0,4 ; 0,6 ; 0.8$ e 1,0; conforme SINGH (1993). O número de descendentes por fêmea foi fixado em 2 , número de machos foi de $2,4,6,8,10,30$ e 50 , sendo que o número de fêmeas foi de $2,4,6,8,10,20$ e 30 .

Foram simulados 50 experimentos para cada combinação de machos $\mathrm{e}$ fềmeas, com os coeficientes de herdabilidade esperados. Para a simulação dos experimentos usou-se o gerador RANNOR do SAS (Statistical Analysis System), que produz desvios aleatórios normais com média zero e variância um. O gerador RANNOR emprega o método de BOX - MULLER (1958), que consiste em considerar a densidade conjunta de duas variáveis aleatórias, $\mathrm{ND}(0,1)$ e o elemento de probabilidade:

$$
f\left(x_{1}, x_{2}\right) d x_{1} d x_{2}=(1 / 2 \pi) \exp \left[-\left(x_{1}^{2}+x_{2}^{2}\right) / 2\right] d x_{1} d_{2}
$$

Convertendo para coordenadas polares $\left(\mathrm{x}_{1}, \mathrm{x}_{2}\right) \rightarrow(\mathrm{r}, \theta)$ e fatorando, tem-se

$$
f(r, \theta) \operatorname{rdrd} \theta=\left\{\exp \left(r^{2} / 2\right) d\left(r^{2} / 2\right)\right\}\{d \theta / 2 \pi\}
$$


A primeira densidade é exponencial na variável $\mathrm{r}^{2} / 2$, enquanto que a segunda é uniforme sobre o intervalo $(0,2 \pi)$. Desvios aleatórios para estas variáveis săo facilmente construidos, fazendo-se

$$
\begin{aligned}
& \theta=2 \pi U_{3}, \mathrm{e} \\
& \mathrm{r}=\left(-2 \log \left(\mathrm{U}^{\prime}\right)\right)^{1 / 2},
\end{aligned}
$$

onde $U, U^{\prime}$ são uniformes $(0,1)$.

Finalmente, fazendo

$$
\mathrm{Y}=\mathrm{R} \cos (\theta)=\left(-2 \log \left(\mathrm{U}^{\prime}\right)\right)^{1 / 2} \cos (2 \pi \mathrm{U})
$$

Y tem distribuição Normal $(0,1)$.

Para cada caso, combinação de machos e fềmeas e, para cada coeficiente de herdabilidade esperado, foram feitas 50 análises de variâncias. De cada uma das análises, foram calculadas para a variável aleatória $\mathrm{H}^{2}$, a sua estimativa $\mathrm{h}^{2}$, média, variância pelas fórmulas aproximadas, médias das variâncias, máximo e minimo e as frequências de se obter coeficientes de herdabilidade negativos, devido ao método (momentos) empregado para estimar os componentes de variância. As estimativas negativas dos componentes de variância foram consideradas nulas. 


\section{RESULTADOS E DISCUSSÃO}

Na tabela 1 a seguir, estão apresentados para cada estatistica, 0 minimo, o máximo e a média de 50 experimentos, para várias combinações de machos e fềmeas com 2 descendentes. Assim, caso = IJ, significa: número de machos e fềmeas, respectivamente.

Tabela 1. Estimativa do coeficiente de herdabilidade (H2S) e variância do coeficiente de herdabilidade, estimada pelas fórmulas de Falconer (VH2SF), Dickerson (VH2SD) e Osborne $\varepsilon$ Paterson, para diferentes coeficientes de herdabilidade esperados (H2).

H2 Caso NObs Estatistica Mínimo Máximo Média

\begin{tabular}{|c|c|c|c|c|c|c|}
\hline \multirow[t]{12}{*}{0.0} & \multirow[t]{4}{*}{22} & \multirow[t]{4}{*}{8} & $\mathrm{H} 2 \mathrm{~S}$ & 0.0000 & 3.0366 & 0.4474 \\
\hline & & & VH2SF & 0.0000 & 12.1463 & 1.7898 \\
\hline & & & VH2SD & 0.0005 & 7.8922 & 1.5407 \\
\hline & & & VH2SOP & 0.0005 & 2.5078 & 0.9316 \\
\hline & \multirow[t]{4}{*}{24} & \multirow[t]{4}{*}{16} & $\mathrm{H} 2 \mathrm{~S}$ & 0.0000 & 2.8394 & 0.3241 \\
\hline & & & VH2SF & 0.0000 & 5.6787 & 0.648 \\
\hline & & & VH2SD & 0.0110 & 6.2400 & 0.5747 \\
\hline & & & VH2SOP & 0.0110 & 1.0662 & 0.279 \\
\hline & \multirow[t]{4}{*}{26} & \multirow[t]{4}{*}{24} & $\mathrm{H} 2 \mathrm{~S}$ & 0.0000 & 0.8603 & 0.138 \\
\hline & & & VH2SF & 0.0000 & 1.1471 & 0.184 \\
\hline & & & $\mathrm{VH} 2 \mathrm{SD}$ & 0.0029 & 0.7978 & 0.130 \\
\hline & & & VH2SOP & 0.0029 & 0.4946 & 0.104 \\
\hline
\end{tabular}


Tabela 1. Estimativa do coeficiente de herdabilidade (H2S) e variância do coeficiente de herdabilidade, estimada pelas fórmulas de Falconer (VH2SF), Dickerson (VH2SD) e Osborne \& Paterson, para diferentes coeficientes de herdabilidade esperados (H2).

\begin{tabular}{ccccccc}
\hline H2 & Caso & NObs & Estatística & Mínimo & Máximo & Média \\
\hline 0,0 & 28 & 32 & H2S & 0.0000 & 1.6507 & 0.1831 \\
& & & VH2SF & 0.0000 & 1.6507 & 0.1831 \\
& & & VH2SD & 0.0028 & 2.1265 & 0.1672 \\
& & & VH2SOP & 0.0028 & 0.7373 & 0.1037
\end{tabular}

\begin{tabular}{|c|c|c|c|c|}
\hline 42 & $\mathrm{H} 2 \mathrm{~S}$ & 0.0000 & 1.3563 & 0.2479 \\
\hline & $\mathrm{VH} 2 \mathrm{SF}$ & 0.0000 & 2.7126 & 0.4958 \\
\hline & VH2SD & 0.0314 & 2.6834 & 0.6949 \\
\hline & $\mathrm{VH} 2 \mathrm{SO}$ & 0.0298 & 16038 & 05 \\
\hline
\end{tabular}

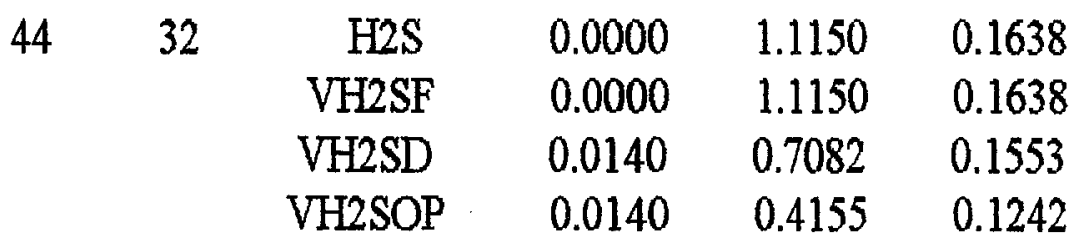

\begin{tabular}{|c|c|c|c|c|}
\hline 48 & $\mathrm{H} 2 \mathrm{~S}$ & 0.0000 & 0.9305 & 0.1361 \\
\hline & $\mathrm{VH} 2 \mathrm{SF}$ & 0.0000 & 0.6203 & 0.09 \\
\hline & VH2SD & 0.0044 & 0.6301 & 0913 \\
\hline & & 0.0044 & 0.3737 & \\
\hline
\end{tabular}

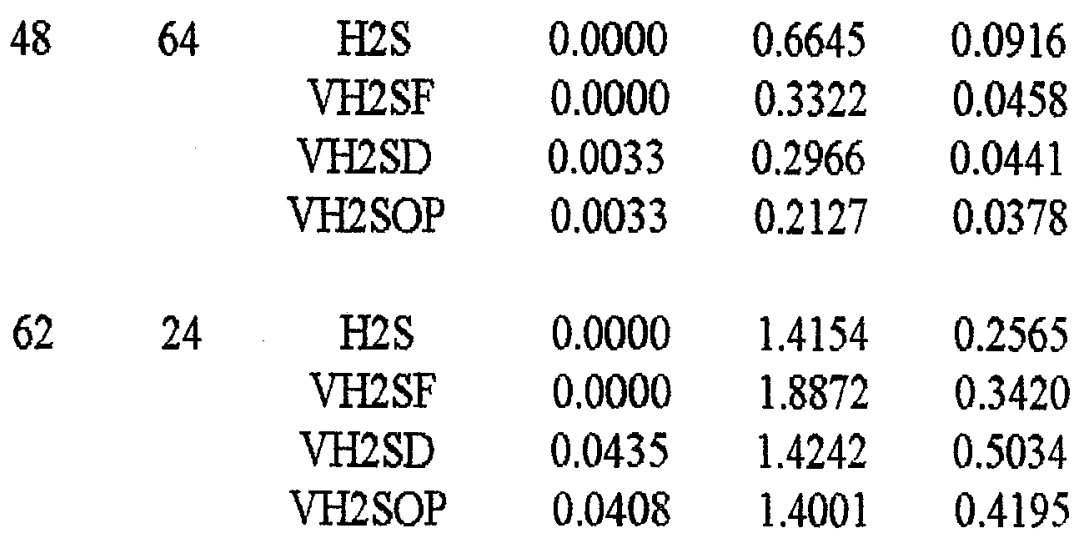


Tabela 1. Estimativa do coeficiente de herdabilidade (H2S) e variância do coeficiente de herdabilidade, estimada pelas formulas de Falconer (VH2SF), Dickerson (VH2SD) e Osbome \& Paterson, para diferentes coeficientes de herdabilidade esperados $(\mathrm{H} 2)$.

\begin{tabular}{ccccccc}
\hline H2 & Caso & NObs & Estatistica & Minimo & Máximo & Média \\
\hline 0,0 & 64 & 48 & H2S & 0.0000 & 1.1429 & 0.1010 \\
& & & VH2SF & 0.0000 & 0.7619 & 0.0673 \\
& & & VH2SD & 0.0145 & 0.6600 & 0.1033 \\
& & VH2SOP & 0.0145 & 0.3399 & 0.0872 \\
& & & & & \\
& \multirow{2}{*}{66} & 72 & H2S & 0.0000 & 0.7397 & 0.0793 \\
& & VH2SF & 0.0000 & 0.3288 & 0.0353 \\
& & VH2SD & 0.0039 & 0.2944 & 0.0456 \\
& & VH2SOP & 0.0039 & 0.1967 & 0.0413
\end{tabular}

$\begin{array}{cccccc}68 & 96 & \text { H2S } & 0.0000 & 0.5655 & 0.0601 \\ & & \text { VH2SF } & 0.0000 & 0.1885 & 0.0200 \\ & \text { VH2SD } & 0.0018 & 0.1772 & 0.0242 \\ & & \text { VH2SOP } & 0.0018 & 0.1312 & 0.0215\end{array}$

\begin{tabular}{|c|c|c|c|c|c|}
\hline 82 & 32 & $\mathrm{H} 2 \mathrm{~S}$ & 0.0000 & 1.8834 & 0.2690 \\
\hline & & VH2SF & 0.0000 & 1.8834 & 0.2690 \\
\hline & & $\mathrm{VH} 2 \mathrm{SD}$ & 0.0275 & 1.1804 & 0.423 \\
\hline & & VH2SOP & 0.0275 & 0.8501 & 0.34 \\
\hline
\end{tabular}

\begin{tabular}{|c|c|c|c|c|}
\hline 84 & $\mathrm{H} 2 \mathrm{~S}$ & 0.0000 & 0.7856 & 0.1020 \\
\hline & VH2SF & 0.0000 & 0.3928 & 0.0510 \\
\hline & VH2SD & 0.0122 & 0.3257 & 0.0721 \\
\hline & VH2SOP & 0.0122 & 02122 & 0.0646 \\
\hline
\end{tabular}

\begin{tabular}{|c|c|c|c|c|}
\hline 86 & $\mathrm{H} 2 \mathrm{~S}$ & 0.0000 & 0.6008 & 0.0561 \\
\hline & VH2SF & 0.0000 & 0.2003 & 0.0187 \\
\hline & VH2SD & 0.0024 & 0.1757 & 0.0293 \\
\hline & VH2SOP & 0.0024 & 0.1276 & 0.0269 \\
\hline
\end{tabular}


Tabela 1. Estimativa do coeficiente de herdabilidade (H2S) e variância do coeficiente de herdabilidade, estimada pelas fórmulas de Falconer (VH2SF), Dickerson (VH2SD) e Osborne \& Paterson, para diferentes coeficientes de herdabilidade esperados (H2).

\begin{tabular}{ccccccc}
\hline H2 & Caso & NObs & Estatistica & Minimo & Máximo & Média \\
\hline 1,0 & 88 & \multirow{2}{*}{128} & H2S & 0.0000 & 0.5235 & 0.0632 \\
& & & VH2SF & 0.0000 & 0.1309 & 0.0158 \\
& & & VH2SD & 0.0030 & 0.1219 & 0.0199 \\
& & & VH2SOP & 0.0030 & 0.0925 & 0.0181
\end{tabular}

\begin{tabular}{|c|c|c|c|c|c|}
\hline 22 & 8 & $\mathrm{H} 2 \mathrm{~S}$ & 0.0000 & 2.8899 & 0.4157 \\
\hline & & VH2SF & 0.0000 & 11.5596 & 1.6628 \\
\hline & & $\mathrm{VH} 2 \mathrm{SD}$ & 0.0030 & 5.5898 & 1.3617 \\
\hline & & VH2SOP & 0.0030 & 3.2012 & 0.9319 \\
\hline 24 & 16 & $\mathrm{H} 2 \mathrm{~S}$ & 0.0000 & 1.8714 & 0.2824 \\
\hline & & VH2SF & 0.0000 & 3.7428 & 0.5647 \\
\hline & & VH2SD & 0.0152 & 2.8019 & 0.4168 \\
\hline & & VH2SOP & 0.0152 & 1.0497 & 0.2709 \\
\hline 26 & 24 & $\mathrm{H} 2 \mathrm{~S}$ & 0.0000 & 1.8578 & 0.1798 \\
\hline & & $\mathrm{VH} 2 \mathrm{SF}$ & 0.0000 & 2.4771 & 0.2397 \\
\hline & & VH2SD & 0.0011 & 2.8408 & 0.2334 \\
\hline & & VH2SOP & 0.0011 & 0.8211 & 0.1442 \\
\hline
\end{tabular}

$\begin{array}{cccccc}28 & 32 & \text { H2S } & 0.0000 & 0.4866 & 0.0474 \\ & & \text { VH2SF } & 0.0000 & 0.4866 & 0.0474 \\ & & \text { VH2SD } & 0.0013 & 0.2890 & 0.0390 \\ & & \text { VH2SOP } & 0.0013 & 0.2239 & 0.0352 \\ & & & & & \\ 42 & 16 & \text { H2S } & 0.0000 & 2.0259 & 0.3668 \\ & & \text { VH2SF } & 0.0000 & 4.0518 & 0.7335 \\ & & \text { VH2SD } & 0.0536 & 2.3820 & 0.7919 \\ & & \text { VH2SOP } & 0.0536 & 1.8694 & 0.6015\end{array}$


Tabela 1. Estimativa do coeficiente de herdabilidade (H2S) e variância do coeficiente de herdabilidade, estimada pelas fórmulas de Falconer (VH2SF), Dickerson (VH2SD) e Osborne \& Paterson, para diferentes coeficientes de herdabilidade esperados (H2).

\begin{tabular}{ccccccc}
\hline H2 & Caso & NObs & Estatística & Mínimo & Máximo & Média \\
\hline 0,2 & 44 & 32 & H2S & 0.0000 & 1.3327 & 0.1272 \\
& & & VH2SF & 0.0000 & 1.3327 & 0.1272 \\
& & & VH2SD & 0.0042 & 1.0939 & 0.1342 \\
& & VH2SOP & 0.0042 & 0.4909 & 0.1086 \\
& & & & & \\
& 46 & 48 & H2S & 0.0000 & 0.7724 & 0.1083 \\
& & VH2SF & 0.0000 & 0.5150 & 0.0722 \\
& & VH2SD & 0.0034 & 0.4568 & 0.0735 \\
& & VH2SOP & 0.0034 & 0.3019 & 0.0607
\end{tabular}

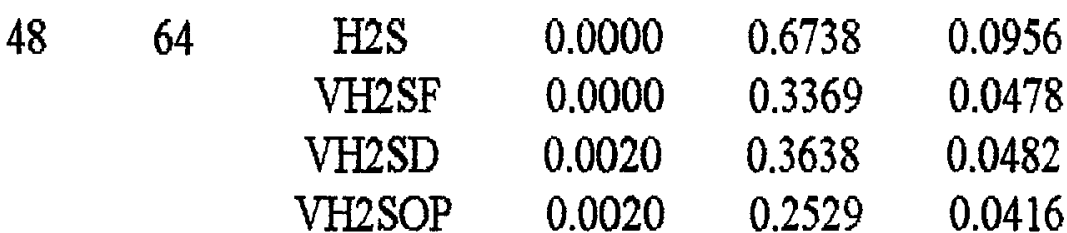

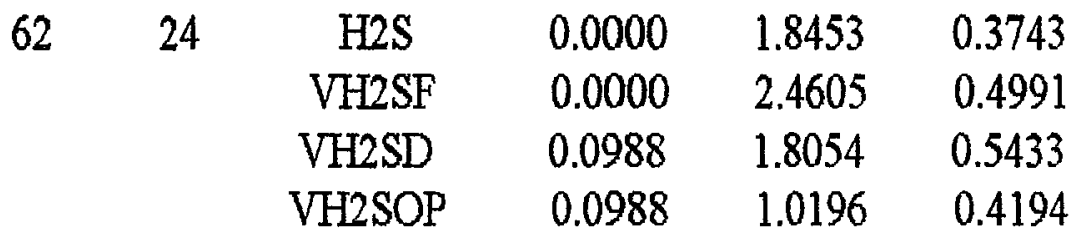

\begin{tabular}{|c|c|c|c|c|c|}
\hline \multirow[t]{4}{*}{64} & \multirow[t]{4}{*}{48} & $\mathrm{H} 2 \mathrm{~S}$ & 0.0000 & 0.9025 & 0.1282 \\
\hline & & VH2SF & 0.0000 & 0.6017 & 0.085 \\
\hline & & $\mathrm{VH} 2 \mathrm{SD}$ & 0.0148 & 0.5042 & 0.10 \\
\hline & & VH2SOP & 0.0148 & 0.3051 & 0.08 \\
\hline \multirow[t]{4}{*}{66} & 72 & $\mathrm{H} 2 \mathrm{~S}$ & 0.0000 & 0.4870 & 0.06 \\
\hline & & $\mathrm{VH} 2 \mathrm{SF}$ & 0.0000 & 0.2165 & \\
\hline & & $\mathrm{VH} 2 \mathrm{SD}$ & 0.0038 & 0.1673 & \\
\hline & & $\mathrm{VH} 2 \mathrm{SOP}$ & 0.0038 & 0.1332 & 0.0 \\
\hline
\end{tabular}


Tabela 1. Estimativa do coeficiente de herdabilidade (H2S) e variância do coeficiente de herdabilidade, estimada pelas fórmulas de Falconer (VH2SF), Dickerson (VH2SD) e Osborne $\&$ Paterson, para diferentes coeficientes de herdabilidade esperados (H2).

H2 Caso NObs Estatistica Minimo Máximo Média

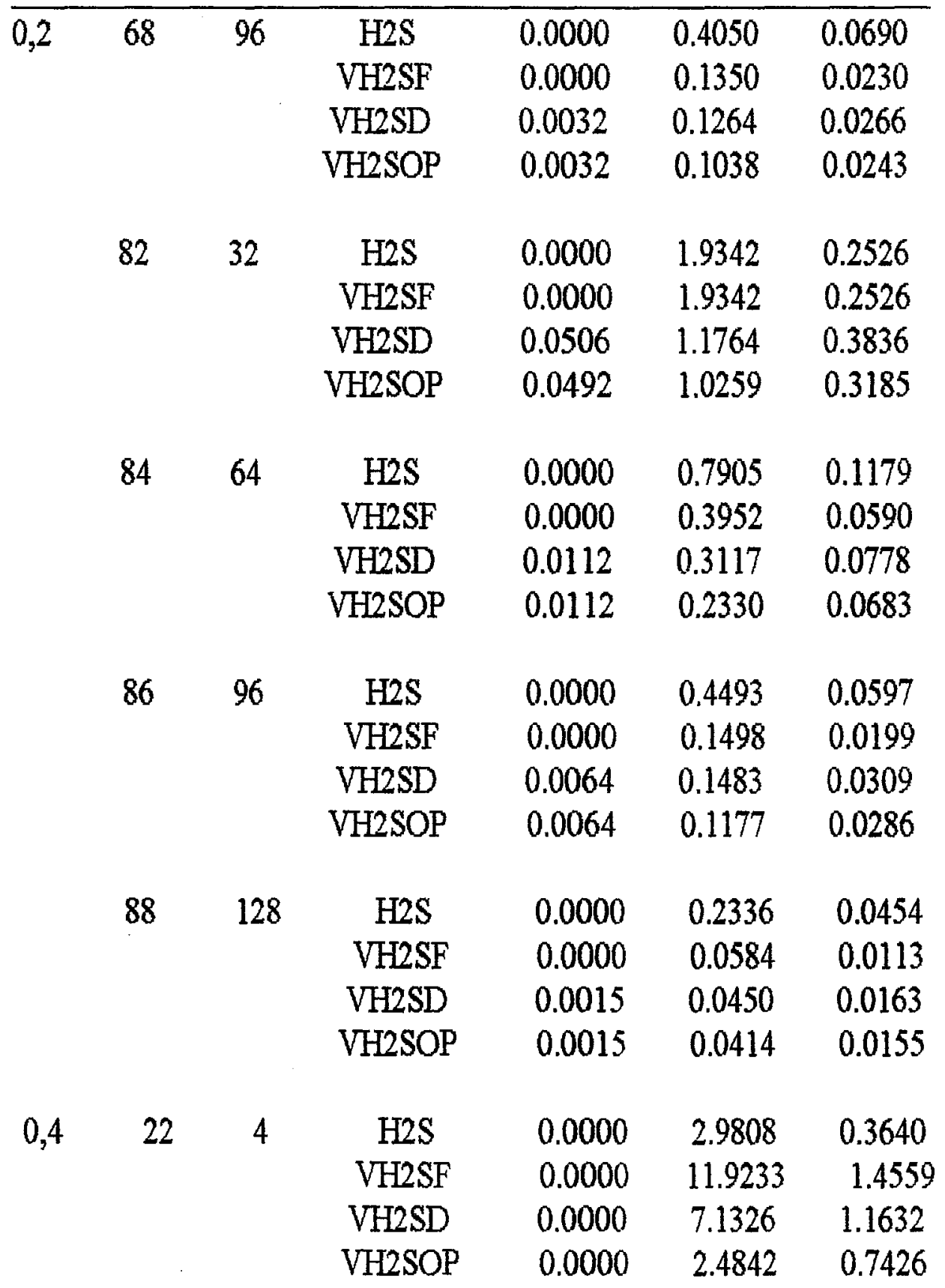


Tabela 1. Estimativa do coeficiente de herdabilidade (H2S) e variância do coeficiente de herdabilidade, estimada pelas formulas de Falconer (VH2SF), Dickerson (VH2SD) e Osborne \& Paterson, para diferentes coeficientes de herdabilidade esperados $(\mathrm{H} 2)$.

H2 Caso NObs Estatistica Mínimo Máximo Média

\begin{tabular}{llccccc}
\hline 0,4 & 24 & 16 & H2S & 0.0000 & 1.6021 & 0.2578 \\
& & H2SF & 0.0000 & 3.2041 & 0.5156 \\
& & VH2SD & 0.0009 & 2.1304 & 0.3892 \\
& & VH2SOP & 0.0009 & 0.9180 & 0.2546 \\
& & & & & \\
& \multirow{2}{*}{26} & 24 & H2S & 0.0000 & 1.0864 & 0.1303 \\
& & VH2SF & 0.0000 & 1.4485 & 0.1738 \\
& & VH2SD & 0.0030 & 1.2254 & 0.1435 \\
& & VH2SOP & 0.0030 & 0.6550 & 0.1066
\end{tabular}

$\begin{array}{llclll}28 & 32 & \text { H2S } & 0.0000 & 0.9460 & 0.1187 \\ & \text { VH2SF } & 0.0000 & 0.9460 & 0.1187 \\ & \text { VH2SD } & 0.0022 & 1.0277 & 0.1038 \\ & & \text { VH2SOP } & 0.0022 & 0.6035 & 0.0745\end{array}$

\begin{tabular}{|c|c|c|c|c|}
\hline 16 & $\mathrm{H} 2 \mathrm{~S}$ & 0.0000 & 2.6575 & 0.3340 \\
\hline & $\mathrm{VH} 2 \mathrm{SF}$ & 0.0000 & 5.3150 & \\
\hline & $\mathrm{VH} 2 \mathrm{SD}$ & 0.0679 & 3.1255 & 0.8 \\
\hline & VH2SOP & 0.0618 & 1.5741 & \\
\hline
\end{tabular}

$\begin{array}{cccccc}44 & 32 & \text { H2S } & 0.0000 & 0.8798 & 0.1196 \\ & & \text { VH2SF } & 0.0000 & 0.8798 & 0.1196 \\ & & \text { VH2SD } & 0.0050 & 0.3848 & 0.1269 \\ & & \text { VH2SOP } & 0.0050 & 0.2936 & 0.1128 \\ & & & & & \\ 46 & 48 & \text { H2S } & 0.0000 & 0.7390 & 0.0920 \\ & & \text { VH2SF } & 0.0000 & 0.4926 & 0.0613 \\ & & \text { VH2SD } & 0.0012 & 0.3573 & 0.0761 \\ & & \text { VH2SOP } & 0.0012 & 0.2386 & 0.0675\end{array}$


Tabela 1. Estimativa do coeficiente de herdabilidade (H2S) e variância do coeficiente de herdabilidade, estimada pelas fórmulas de Falconer (VH2SF), Dickerson (VH2SD) e Osborne \& Paterson, para diferentes coeficientes de herdabilidade esperados (H2).

\begin{tabular}{llllll}
\hline H2 & Caso N Obs Estatistica & Minimo & Máximo & Média
\end{tabular}

\begin{tabular}{|c|c|c|c|c|c|c|}
\hline 0,4 & 48 & 64 & $\mathrm{H} 2 \mathrm{~S}$ & 0.0000 & 0.5639 & 0.0833 \\
\hline & & & $\mathrm{VH} 2 \mathrm{SF}$ & 0.0000 & 0.2819 & 0.0417 \\
\hline & & & $\mathrm{VH} 2 \mathrm{SD}$ & 0.0020 & 0.2368 & 0.0386 \\
\hline & & & VH2SOP & 0.0020 & 0.1755 & 0.0346 \\
\hline & 62 & 24 & $\mathrm{H} 2 \mathrm{~S}$ & 0.0000 & 1.6938 & 0.3073 \\
\hline & & & $\mathrm{VH} 2 \mathrm{SF}$ & 0.0000 & 2.2583 & 0.4098 \\
\hline & & & VHzSD & 0.0638 & 1.5363 & 0.5572 \\
\hline & & & VH2SOP & 0.0638 & 1.4686 & 0.4605 \\
\hline & 64 & 48 & $\mathrm{H} 2 \mathrm{~S}$ & 0.0000 & 0.7513 & 0.1206 \\
\hline & & & VH2SF & 0.0000 & 0.5009 & 0.0804 \\
\hline & & & VH2SD & 0.0148 & 0.3302 & 0.1088 \\
\hline & & & VH2SOP & 0.0148 & 0.2633 & 0.0960 \\
\hline & 66 & 72 & $\mathrm{H} 2 \mathrm{~S}$ & 0.0000 & 0.3586 & 0.0529 \\
\hline & & & VH2SF & 0.0000 & 0.1594 & 0.0235 \\
\hline & & & VH2SD & 0.0044 & 0.1422 & 0.0368 \\
\hline & & & $\mathrm{VH} 2 \mathrm{SOP}$ & 0.0044 & 0.1185 & 0.0347 \\
\hline & 68 & 96 & $\mathrm{H} 2 \mathrm{~S}$ & 0.0000 & 0.6572 & 0.0794 \\
\hline & & & $\mathrm{VH} 2 \mathrm{SF}$ & 0.0000 & 0.2191 & 0.0265 \\
\hline & & & $\mathrm{VH} 2 \mathrm{SD}$ & 0.0021 & 0.2075 & 0.0301 \\
\hline & & & VH2SOP & 0.0021 & 0.1455 & 0.0269 \\
\hline & 82 & 32 & $\mathrm{H} 2 \mathrm{~S}$ & 0.0000 & 1.2640 & 0.2613 \\
\hline & & & VH2SF & 0.0000 & 1.2640 & 0.2613 \\
\hline & & & $\mathrm{VH} 2 \mathrm{SD}$ & 0.0296 & 1.0136 & 0.4295 \\
\hline & & & VH2SOP & 0.0296 & 0.8992 & 0.3630 \\
\hline
\end{tabular}


Tabela 1. Estimativa do coeficiente de herdabilidade (H2S) e variância do coeficiente de herdabilidade, estimada pelas fórmulas de Falconer (VH2SF), Dickerson (VH2SD) e Osborne \& Paterson, para diferentes coeficientes de herdabilidade esperados (H2).

H2 Caso NObs Estatistica Minimo Máximo Média

\begin{tabular}{|c|c|c|c|c|c|c|}
\hline \multirow[t]{12}{*}{0,4} & \multirow[t]{4}{*}{84} & \multirow[t]{4}{*}{64} & $\mathrm{H} 2 \mathrm{~S}$ & 0.0000 & 1.0163 & 0.1090 \\
\hline & & & VH2SF & 0.0000 & 0.5081 & 0.0545 \\
\hline & & & $\mathrm{VH} 2 \mathrm{SD}$ & 0.0065 & 0.4487 & 0.0820 \\
\hline & & & VH2SOP & 0.0065 & 0.2520 & 0.0718 \\
\hline & \multirow[t]{4}{*}{86} & \multirow[t]{4}{*}{96} & $\mathrm{H} 2 \mathrm{~S}$ & 0.0000 & 0.4787 & 0.0686 \\
\hline & & & $\mathrm{VH} 2 \mathrm{SF}$ & 0.0000 & 0.1596 & 0.0229 \\
\hline & & & VH2SD & 0.0054 & 0.1580 & 0.0331 \\
\hline & & & VH2SOP & 0.0054 & 0.1231 & 0.0306 \\
\hline & \multirow[t]{4}{*}{88} & \multirow[t]{4}{*}{128} & $\mathrm{H} 2 \mathrm{~S}$ & 0.0000 & 0.3534 & 0.0575 \\
\hline & & & $\mathrm{VH} 2 \mathrm{SF}$ & 0.0000 & 0.0883 & 0.0144 \\
\hline & & & $\mathrm{VH} 2 \mathrm{SD}$ & 0.0020 & 0.0708 & 0.0177 \\
\hline & & & VH2SOP & 0.0020 & 0.0610 & 0.0164 \\
\hline
\end{tabular}

$\begin{array}{ccccccc}0,6 & 22 & 8 & \text { H2S } & 0.0000 & 1.8531 & 0.3251 \\ & & \text { VH2SF } & 0.0000 & 7.4126 & 1.3005 \\ & & \text { VH2SD } & 0.0030 & 5.3066 & 1.2811 \\ & & \text { VH2SOP } & 0.0029 & 4.3090 & 0.9458\end{array}$

\begin{tabular}{|c|c|c|c|c|}
\hline 24 & $\mathrm{H} 2 \mathrm{~S}$ & 0.0000 & 1.7230 & 0.2427 \\
\hline & VH2SF & 0.0000 & 3.4460 & 0.4855 \\
\hline & VH2SD & 0.0080 & 2.9327 & 0.3529 \\
\hline & VH2SOP & 0,0080 & 0.9620 & 0.2204 \\
\hline
\end{tabular}

\begin{tabular}{|c|c|c|c|c|}
\hline 26 & $\mathrm{H} 2 \mathrm{~S}$ & 0.0000 & 1.4499 & 0.1334 \\
\hline & VH2SF & 0.0000 & 1.9332 & 0.1779 \\
\hline & VH2SD & 0.0023 & 1.9195 & 0.1518 \\
\hline & VH2SOP & 0.0023 & 0.7862 & 0.1035 \\
\hline
\end{tabular}


Tabela 1. Estimativa do coeficiente de herdabilidade (H2S) e variância do coeficiente de herdabilidade, estimada pelas fórmulas de Falconer (VH2SF), Dickerson (VH2SD) e Osborne \& Paterson, para diferentes coeficientes de herdabilidade esperados (H2).

\begin{tabular}{ccccccc}
\hline H2 & Caso & NObs & Estatistica & Minimo & Máximo & Média \\
\hline 0,6 & 28 & 32 & H2S & 0.0000 & 1.2900 & 0.0639 \\
& & & VH2SF & 0.0000 & 1.2900 & 0.0639 \\
& & & VH2SD & 0.0007 & 1.4528 & 0.0701 \\
& & & VH2SOP & 0.0007 & 0.6706 & 0.0488 \\
& & & & & & \\
& \multirow{2}{*}{42} & 16 & H2S & 0.0000 & 1.7847 & 0.3710 \\
& & & VH2SF & 0.0000 & 3.5693 & 0.7419 \\
& & & VH2SD & 0.0099 & 2.2177 & 0.6544 \\
& & & VH2SOP & 0.0099 & 1.3441 & 0.4978
\end{tabular}

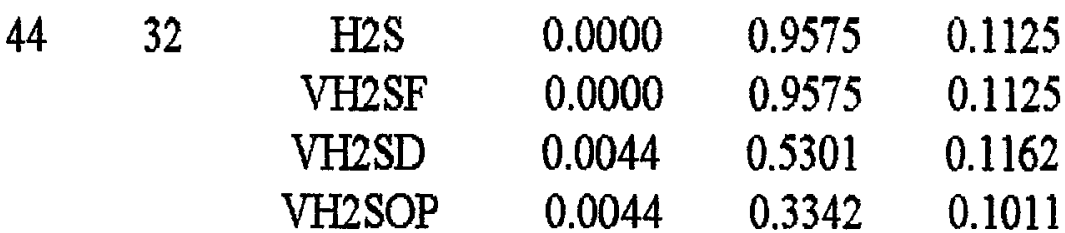

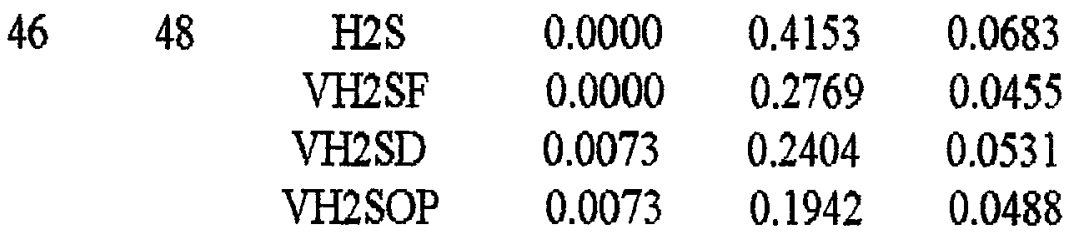

$\begin{array}{cccccc}48 & 64 & \text { H2S } & 0.0000 & 0.3840 & 0.0601 \\ & & \text { VH2SF } & 0.0000 & 0.1920 & 0.0300 \\ & & \text { VH2SD } & 0.0008 & 0.1676 & 0.0333 \\ & & \text { VH2SOP } & 0.0008 & 0.1418 & 0.0306 \\ & & & & & \\ 62 & 24 & \text { H2S } & 0.0000 & 2.2859 & 0.2956 \\ & & \text { VH2SF } & 0.0000 & 3.0478 & 0.3941 \\ & & \text { VH2SD } & 0.0400 & 2.0999 & 0.6028 \\ & & \text { VH2SOP } & 0.0400 & 1.0794 & 0.4820\end{array}$


Tabela 1. Estimativa do coeficiente de herdabilidade (H2S) e variância do coeficiente de herdabilidade, estimada pelas fórmulas de Falconer (VH2SF), Dickerson (VH2SD) e Osborne $\varepsilon$ Paterson, para diferentes coeficientes de herdabilidade esperados $(\mathrm{H} 2)$.

\begin{tabular}{ccccccc}
\hline H2 & Caso & NObs & Estatística & Mínimo & Máximo & Média \\
\hline 0,6 & 64 & 48 & H2S & 0.0000 & 0.8243 & 0.1292 \\
& & & VH2SF & 0.0000 & 0.5495 & 0.0861 \\
& & & VH2SD & 0.0069 & 0.4472 & 0.1094 \\
& & & VH2SOP & 0.0069 & 0.2844 & 0.0957
\end{tabular}

\begin{tabular}{|c|c|c|c|c|c|}
\hline \multirow[t]{4}{*}{66} & \multirow[t]{4}{*}{72} & $\mathrm{H} 2 \mathrm{~S}$ & 0.0000 & 0.4216 & 0.0537 \\
\hline & & VH2SF & 0.0000 & 0.1874 & 0.0238 \\
\hline & & $\mathrm{VH} 2 \mathrm{SD}$ & 0.0035 & 0.1540 & 0.0367 \\
\hline & & VH2SOP & 0.0035 & 0.1239 & 0.0344 \\
\hline \multirow[t]{4}{*}{68} & \multirow[t]{4}{*}{96} & $\mathrm{H} 2 \mathrm{~S}$ & 0.0000 & 0.4652 & 0.0706 \\
\hline & & VH2SF & 0.0000 & 0.1551 & 0.0235 \\
\hline & & VH2SD & 0.0033 & 0.1353 & 0.0246 \\
\hline & & VH2SOP & 0.0033 & 0.1061 & 0.0227 \\
\hline \multirow[t]{4}{*}{82} & \multirow[t]{4}{*}{32} & $\mathrm{H} 2 \mathrm{~S}$ & 0.0000 & 2.0678 & 0.3096 \\
\hline & & VH2SF & 0.0000 & 2.0678 & 0.3096 \\
\hline & & VH2SD & 0.0492 & 1.4783 & 0.4036 \\
\hline & & VH2SOP & 0.0492 & 0.8206 & 0.3164 \\
\hline
\end{tabular}

$\begin{array}{lccccc}84 & 64 & \text { H2S } & 0.0000 & 0.6413 & 0.0932 \\ & \text { VH2SF } & 0.0000 & 0.3207 & 0.0466 \\ & \text { VH2SD } & 0.0103 & 0.2708 & 0.0706 \\ & & \text { VH2SOP } & 0.0103 & 0.2127 & 0.0648\end{array}$

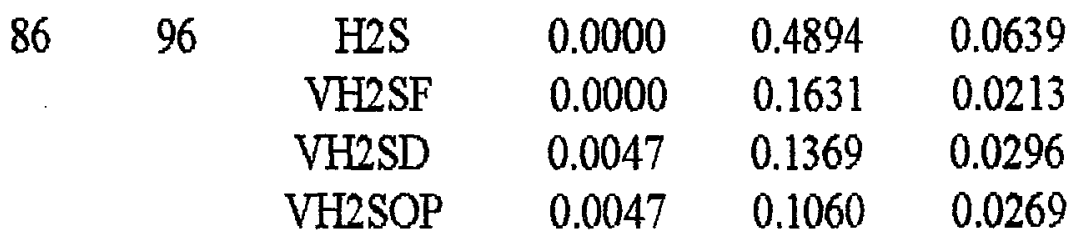


Tabela 1. Estimativa do coeficiente de herdabilidade (H2S) e variância do coeficiente de herdabilidade, estimada pelas fórmulas de Falconer (VH2SF), Dickerson (VH2SD) e Osborne \& Paterson, para diferentes coeficientes de herdabilidade esperados (H2).

\begin{tabular}{lcccccc}
\hline H2 & Caso & NObs & Estatistica & Mínimo & Máximo & Média \\
\hline 0,6 & 88 & 128 & H2S & 0.0000 & 0.3120 & 0.0571 \\
& & & VH2SF & 0.0000 & 0.0780 & 0.0143 \\
& & & VH2SD & 0.0021 & 0.0660 & 0.0193 \\
& & & VH2SOP & 0.0021 & 0.0563 & 0.0183
\end{tabular}

\begin{tabular}{|c|c|c|c|c|c|}
\hline 0,8 & 8 & $\mathrm{H} 2 \mathrm{~S}$ & 0.0000 & 2.9706 & 0.3616 \\
\hline & & $\mathrm{VH} 2 \mathrm{SF}$ & 0.0000 & 11.8824 & 1.4464 \\
\hline & & VH2SD & 0.0179 & 6.3150 & 1.3357 \\
\hline & & VH2SOP & 0.0179 & 2.7987 & 0.8239 \\
\hline 24 & 16 & $\mathrm{H} 2 \mathrm{~S}$ & 0.0000 & 1.3974 & 0.2275 \\
\hline & & VH2SF & 0.0000 & 2.7949 & 0.4550 \\
\hline & & VH2SD & 0.0080 & 2.1747 & 0.4004 \\
\hline & & VH2SOP & 0.0080 & 0.9976 & 0.2669 \\
\hline 26 & 24 & $\mathrm{H} 2 \mathrm{~S}$ & 0.0000 & 1.0683 & 0.1778 \\
\hline & & $\mathrm{VH} 2 \mathrm{SF}$ & 0.0000 & 1.4243 & 0.2370 \\
\hline & & VH2SD & 0.0016 & 1.0286 & 0.1912 \\
\hline & & VH2SOP & 0.0016 & 0.6708 & 0.1435 \\
\hline 28 & 32 & $\mathrm{H} 2 \mathrm{~S}$ & 0.0000 & 0.9040 & 0.1045 \\
\hline & & $\mathrm{VH} 2 \mathrm{SF}$ & 0.0000 & 0.9040 & 0.1045 \\
\hline & & $\mathrm{VH} 2 \mathrm{SD}$ & 0.0018 & 0.8359 & 0.0831 \\
\hline & & VH2SOP & 0.0018 & 0.5036 & 0.0624 \\
\hline 42 & 16 & $\mathrm{H} 2 \mathrm{~S}$ & 0.0000 & 2.7624 & 0.4518 \\
\hline & & VH2SF & 0.0000 & 5.5248 & 0.9036 \\
\hline & & VH2SD & 0.0947 & 3.9817 & 0.8305 \\
\hline & & VH2SOP & 0.0947 & 1.4575 & 0.5538 \\
\hline
\end{tabular}


Tabela 1. Estimativa do coeficiente de herdabilidade (H2S) e variância do coeficiente de herdabilidade, estimada pelas formulas de Falconer (VH2SF), Dickerson (VH2SD) e Osborne $\varepsilon$ Paterson, para diferentes coeficientes de herdabilidade esperados (H2).

\begin{tabular}{lcccccc}
\hline H2 & Caso & NObs & Estatistica & Mínimo & Máximo & Média \\
\hline 0,8 & 44 & 32 & H2S & 0.0000 & 1.5473 & 0.1367 \\
& & & VH2SF & 0.0000 & 1.5473 & 0.1367 \\
& & & VH2SD & 0.0159 & 1.3396 & 0.1591 \\
& & VH2SOP & 0.0159 & 0.5081 & 0.1222 \\
& & & & & \\
& \multirow{4}{*}{46} & 48 & H2S & 0.0000 & 1.0659 & 0.1116 \\
& & VH2SF & 0.0000 & 0.7106 & 0.0744 \\
& & VH2SD & 0.0041 & 0.7084 & 0.0814 \\
& & & VH2SOP & 0.0041 & 0.3837 & 0.0670
\end{tabular}

\begin{tabular}{|c|c|c|c|c|c|c|}
\hline & 48 & 64 & $\mathrm{H} 2 \mathrm{~S}$ & 0.0000 & 0.4968 & 0.0871 \\
\hline & & & VH2SF & 0.0000 & 0.2484 & 0.0436 \\
\hline & & & VH2SD & 0.0042 & 0.1881 & 0.0413 \\
\hline & & & $\mathrm{VH} 2 \mathrm{SOP}$ & 0.0042 & 0.1480 & 0.0372 \\
\hline & 62 & 24 & $\mathrm{H} 2 \mathrm{~S}$ & 0.0000 & 1.3453 & 0.2373 \\
\hline & & & $\mathrm{VH} 2 \mathrm{SF}$ & 0.0000 & 1.7937 & 0.3164 \\
\hline & & & $\mathrm{VH} 2 \mathrm{SD}$ & 0.0725 & 1.3683 & 0.4795 \\
\hline & & & VH2SOP & 0.0725 & 0.9789 & 0.4136 \\
\hline & 64 & 48 & $\mathrm{H} 2 \mathrm{~S}$ & 0.0000 & 0.6434 & 0.1339 \\
\hline & & & VH2SF & 0.0000 & 0.4289 & 0.0893 \\
\hline & & & VH2SD & 0.0044 & 0.4327 & 0.1064 \\
\hline & & & VH2SOP & 0.0044 & 0.3125 & 0.0927 \\
\hline 0,8 & 66 & 72 & $\mathrm{H} 2 \mathrm{~S}$ & 0.0000 & 0.8870 & 0.0686 \\
\hline & & & VH2SF & 0.0000 & 0.3942 & 0.0305 \\
\hline & & & VH2SD & 0.0029 & 0.4152 & 0.0413 \\
\hline & & & VH2SOP & 0.0029 & 0.2532 & 0.0358 \\
\hline
\end{tabular}


Tabela 1. Estimativa do coeficiente de herdabilidade (H2S) e variância do coeficiente de herdabilidade, estimada pelas fórmulas de Falconer (VH2SF), Dickerson (VH2SD) e Osborne \& Paterson, para diferentes coeficientes de herdabilidade esperados (H2).

\begin{tabular}{|c|c|c|c|c|c|c|}
\hline $\mathrm{H} 2$ & Caso & NObs & Estatística & Mínimo & Máximo & Média \\
\hline \multirow[t]{12}{*}{0,8} & \multirow[t]{4}{*}{68} & \multirow[t]{4}{*}{96} & $\mathrm{H} 2 \mathrm{~S}$ & 0.0000 & 0.3433 & 0.0598 \\
\hline & & & VH2SF & 0.0000 & 0.1144 & 0.0199 \\
\hline & & & $\mathrm{VH} 2 \mathrm{SD}$ & 0.0022 & 0.0999 & 0.0232 \\
\hline & & & VH2SOP & 0.0022 & 0.0838 & 0.0216 \\
\hline & \multirow[t]{4}{*}{82} & \multirow[t]{4}{*}{32} & $\mathrm{H} 2 \mathrm{~S}$ & 0.0000 & 1.2832 & 0.2169 \\
\hline & & & VH2SF & 0.0000 & 1.2832 & 0.2169 \\
\hline & & & VH2SD & 0.0540 & 1.0001 & 0.3823 \\
\hline & & & $\mathrm{VH} 2 \mathrm{SOP}$ & 0.0466 & 0.7474 & 0.3389 \\
\hline & \multirow[t]{4}{*}{84} & \multirow[t]{4}{*}{64} & $\mathrm{H} 2 \mathrm{~S}$ & 0.0000 & 0.5706 & 0.0938 \\
\hline & & & VH2SF & 0.0000 & 0.2853 & 0.0469 \\
\hline & & & VH2SD & 0.0144 & 0.3242 & 0.0734 \\
\hline & & & VH2SOP & 0.0144 & 0.2405 & 0.0663 \\
\hline
\end{tabular}

$\begin{array}{cccccc}86 & 96 & \mathrm{H} 2 \mathrm{~S} & 0.0000 & 0.6241 & 0.0834 \\ & & \text { VH2SF } & 0.0000 & 0.2080 & 0.0278 \\ & \text { VH2SD } & 0.0024 & 0.1827 & 0.0354 \\ & & \text { VH2SOP } & 0.0024 & 0.1308 & 0.0315\end{array}$

$\begin{array}{lccccc}88 & 128 & \text { H2S } & 0.0000 & 0.2534 & 0.0539 \\ & \text { VH2SF } & 0.0000 & 0.0633 & 0.0135 \\ & \text { VH2SD } & 0.0017 & 0.0579 & 0.0197 \\ & \text { VH2SOP } & 0.0017 & 0.0522 & 0.0188\end{array}$

\begin{tabular}{|c|c|c|c|c|c|}
\hline $1,0 \quad 22$ & 8 & $\mathrm{H} 2 \mathrm{~S}$ & 0.0000 & 2.5415 & 0.3627 \\
\hline & & VH2SF & 0.0000 & 10.1661 & 1.45 \\
\hline & & VH2SD & 0.0011 & 5.6639 & $1.07^{\prime}$ \\
\hline & & $\mathrm{VH} 2 \mathrm{SOP}$ & 0.0011 & 22807 & 61 \\
\hline
\end{tabular}


Tabela 1. Estimativa do coeficiente de herdabilidade (H2S) e variância do coeficiente de herdabilidade, estimada pelas fórmulas de Falconer (VH2SF), Dickerson (VH2SD) e Osborne $\varepsilon$ Paterson, para diferentes coeficientes de herdabilidade esperados $(\mathrm{H} 2)$.

\begin{tabular}{ccccccc}
\hline H2 & Caso & NObs & Estatistica & Mínimo & Máximo & Média \\
\hline 1,0 & 24 & 16 & H2S & 0.0000 & 1.9014 & 0.2129 \\
& & & VH2SF & 0.0000 & 3.8029 & 0.4258 \\
& & & VH2SD & 0.0018 & 2.8764 & 0.3922 \\
& & VH2SOP & 0.0018 & 1.0441 & 0.2417 \\
& & & & & \\
& \multirow{2}{*}{26} & 24 & H2S & 0.0000 & 1.4975 & 0.1431 \\
& & VH2SF & 0.0000 & 1.9966 & 0.1908 \\
& & VH2SD & 0.0055 & 1.8394 & 0.1801 \\
& & & VH2SOP & 0.0055 & 0.7266 & 0.1200
\end{tabular}

$\begin{array}{llclll}28 & 32 & \text { H2S } & 0.0000 & 0.8798 & 0.1293 \\ & & \text { VH2SF } & 0.0000 & 0.8798 & 0.1293 \\ & & \text { VH2SD } & 0.0008 & 0.8613 & 0.1058 \\ & & \text { VH2SOP } & 0.0008 & 0.5274 & 0.0827\end{array}$

$\begin{array}{cccccc}42 & 16 & \text { H2S } & 0.0000 & 1.9135 & 0.2979 \\ & & \text { VH2SF } & 0.0000 & 3.8269 & 0.5957 \\ & \text { VH2SD } & 0.0297 & 2.5268 & 0.7264 \\ & & \text { VH2SOP } & 0.0277 & 1.8321 & 0.5669\end{array}$

\begin{tabular}{|c|c|c|c|c|c|}
\hline \multirow[t]{4}{*}{44} & \multirow[t]{4}{*}{32} & $\mathrm{H} 2 \mathrm{~S}$ & 0.0000 & 1.3389 & 0.2195 \\
\hline & & VH2SF & 0.0000 & 1.3389 & 0.2195 \\
\hline & & VH2SD & 0.0027 & 0.9546 & 0.1711 \\
\hline & & VH2SOP & 0.0027 & 0.4252 & 0.1308 \\
\hline \multirow[t]{4}{*}{46} & \multirow[t]{4}{*}{48} & $\mathrm{H} 2 \mathrm{~S}$ & 0.0000 & 0.4795 & 0.1061 \\
\hline & & $\mathrm{VH} 2 \mathrm{SF}$ & 0.0000 & 0.3197 & 0.0707 \\
\hline & & $\mathrm{VH} 2 \mathrm{SD}$ & 0.0028 & 0.2221 & 0.0692 \\
\hline & & VH2SOP & 0.0028 & 0.1828 & 0.0622 \\
\hline
\end{tabular}


Tabela 1. Estimativa do coeficiente de herdabilidade (H2S) e variância do coeficiente de herdabilidade, estimada pelas fórmulas de Falconer (VH2SF), Dickerson (VH2SD) e Osborne $\varepsilon$ Paterson, para diferentes coeficientes de herdabilidade esperados ( $\mathrm{H} 2)$.

H2 Caso NObs Estatistica Minimo Máximo Média

\begin{tabular}{|c|c|c|c|c|c|c|}
\hline \multirow[t]{12}{*}{1,0} & \multirow[t]{4}{*}{48} & \multirow[t]{4}{*}{64} & $\mathrm{H} 2 \mathrm{~S}$ & 0.0000 & 0.5331 & 0.0783 \\
\hline & & & VH2SF & 0.0000 & 0.2665 & 0.0391 \\
\hline & & & VH2SD & 0.0020 & 0.2127 & 0.0412 \\
\hline & & & VH2SOP & 0.0020 & 0.1666 & 0.0369 \\
\hline & \multirow[t]{4}{*}{62} & \multirow[t]{4}{*}{24} & $\mathrm{H} 2 \mathrm{~S}$ & 0.0000 & 1.6269 & 0.2455 \\
\hline & & & $\mathrm{VH} 2 \mathrm{SF}$ & 0.0000 & 2.1691 & 0.3273 \\
\hline & & & $\mathrm{VH} 2 \mathrm{SD}$ & 0.0110 & 1.6576 & 0.5165 \\
\hline & & & VH2SOP & 0.0108 & 0.9943 & 0.4297 \\
\hline & \multirow[t]{4}{*}{64} & \multirow[t]{4}{*}{48} & $\mathrm{H} 2 \mathrm{~S}$ & 0.0000 & 0.6455 & 0.1126 \\
\hline & & & $\mathrm{VH} 2 \mathrm{SF}$ & 0.0000 & 0.4303 & 0.0751 \\
\hline & & & $\mathrm{VH} 2 \mathrm{SD}$ & 0.0060 & 0.3321 & 0.0988 \\
\hline & & & VH2SOP & 0.0060 & 0.2362 & 0.0882 \\
\hline
\end{tabular}

$\begin{array}{llllll}66 & 72 & \mathrm{H} 2 \mathrm{~S} & 0.0000 & 0.5402 & 0.0778\end{array}$

$\begin{array}{llll}\mathrm{VH} 2 \mathrm{SF} & 0.0000 & 0.2401 & 0.0346\end{array}$

$\begin{array}{llll}\text { VH2SD } & 0.0057 & 0.1986 & 0.0385\end{array}$

$\begin{array}{llll}\mathrm{VH} 2 \mathrm{SOP} & 0.0057 & 0.1545 & 0.0349\end{array}$

$\begin{array}{cccccc}68 & 96 & \text { H2S } & 0.0000 & 0.5048 & 0.0784 \\ & & \text { VH2SF } & 0.0000 & 0.1683 & 0.0261 \\ & \text { VH2SD } & 0.0032 & 0.1572 & 0.0300 \\ & & \text { VH2SOP } & 0.0032 & 0.1206 & 0.0270\end{array}$

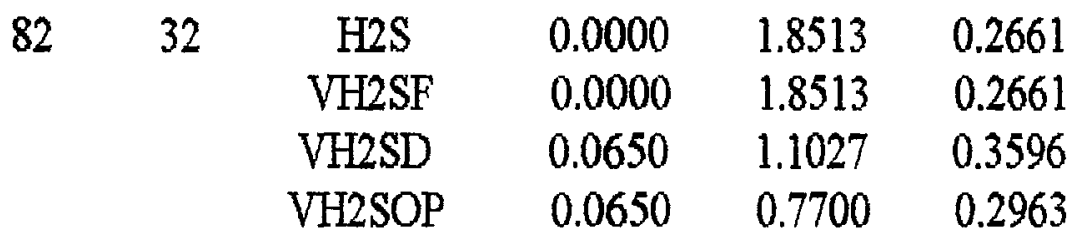


Tabela 1. Estimativa do coeficiente de herdabilidade (H2S) e variância do coeficiente de herdabilidade, estimada pelas formulas de Falconer (VH2SF), Dickerson (VH2SD) e Osborne $\varepsilon$ Paterson, para diferentes coeficientes de herdabilidade esperados (H2).

H2 Caso NObs Estatística Mínimo Máximo Média

\begin{tabular}{lllllll}
\hline 1,0 & 84 & 64 & $\mathrm{H} 2 \mathrm{~S}$ & 0.0000 & 0.8135 & 0.1100 \\
& & $\mathrm{VH} 2 \mathrm{SF}$ & 0.0000 & 0.4068 & 0.0550 \\
& & $\mathrm{VH} 2 \mathrm{SD}$ & 0.0081 & 0.3465 & 0.0760 \\
& & $\mathrm{VH} 2 \mathrm{SOP}$ & 0.0081 & 0.2218 & 0.0680
\end{tabular}

\begin{tabular}{|c|c|c|c|c|c|}
\hline 86 & 96 & $\mathrm{H} 2 \mathrm{~S}$ & 0.0000 & 0.3750 & 0.0680 \\
\hline & & $\mathrm{VH} 2 \mathrm{SF}$ & 0.0000 & 0.1250 & 0.0227 \\
\hline & & VH2SD & 0.0035 & 0.0925 & 0.0315 \\
\hline & & VH2SOP & 0.0035 & 0.0787 & 0.0296 \\
\hline 88 & 128 & $\mathrm{H} 2 \mathrm{~S}$ & 0.0000 & 0.3308 & 0.0469 \\
\hline & & VH2SF & 0.0000 & 0.0827 & 0.0117 \\
\hline & & $\mathrm{VH} 2 \mathrm{SD}$ & 0.0029 & 0.0648 & 0.0167 \\
\hline & & VH2SOP & 0.0029 & 0.0547 & 0.0158 \\
\hline 1010 & 200 & $\mathrm{H} 2 \mathrm{~S}$ & 0.0000 & 0.2279 & 0.0283 \\
\hline & & $\mathrm{VH} 2 \mathrm{SF}$ & 0.0000 & 0.0365 & 0.0045 \\
\hline & & $\mathrm{VH} 2 \mathrm{SD}$ & 0.0014 & 0.0344 & 0.0082 \\
\hline & & VH2SOP & 0.0014 & 0.0307 & 0.0080 \\
\hline 1020 & 400 & $\mathrm{H} 2 \mathrm{~S}$ & 0.0000 & 0.1432 & 0.0207 \\
\hline & & $\mathrm{VH} 2 \mathrm{SF}$ & 0.0000 & 0.0115 & 0.0017 \\
\hline & & $\mathrm{VH} 2 \mathrm{SD}$ & 0.0002 & 0.0108 & 0.0023 \\
\hline & & VH2SOP & 0.0002 & 0.0101 & 0.0022 \\
\hline 1030 & 600 & $\mathrm{H} 2 \mathrm{~S}$ & 0.0000 & 0.1318 & 0.0127 \\
\hline & & $\mathrm{VH} 2 \mathrm{SF}$ & 0.0000 & 0.0070 & 0.0007 \\
\hline & & $\mathrm{VH} 2 \mathrm{SD}$ & 0.0001 & 0.0072 & 0.0010 \\
\hline & & VH2SOP & 0.0001 & 0.0068 & 0.0010 \\
\hline
\end{tabular}


Tabela 1. Estimativa do coeficiente de herdabilidade (H2S) e variância do coeficiente de herdabilidade, estimada pelas fórmulas de Falconer (VH2SF), Dickerson (VH2SD) e Osborne $\varepsilon$ Paterson, para diferentes coeficientes de herdabilidade esperados $(\mathrm{H} 2)$.

H2 Caso Nobs Estatistica Minimo Máximo Média

\begin{tabular}{|c|c|c|c|c|c|}
\hline \multirow[t]{4}{*}{$0,0 \quad 3010$} & 600 & $\mathrm{H} 2 \mathrm{~S}$ & 0.0000 & 0.1731 & 0.0191 \\
\hline & & VH2SF & 0.0000 & 0.0092 & 0.0010 \\
\hline & & $\mathrm{VH} 2 \mathrm{SD}$ & 0.0005 & 0.0083 & 0.0028 \\
\hline & & VH2SOP & 0.0005 & 0.0076 & 0.0028 \\
\hline
\end{tabular}

$\begin{array}{cccccc}3020 & 1200 & \text { H2S } & 0.0000 & 0.0660 & 0.0071 \\ & & \text { VH2SF } & 0.0000 & 0.0018 & 0.0002 \\ & & \text { VH2SD } & 0.0002 & 0.0016 & 0.0006 \\ & & \text { VH2SOP } & 0.0002 & 0.0016 & 0.0006\end{array}$

$\begin{array}{cccccc}3030 & 1800 & \text { H2S } & 0.0000 & 0.0614 & 0.0065 \\ & & \text { VH2SF } & 0.0000 & 0.0011 & 0.0001 \\ & \text { VH2SD } & 0.0001 & 0.0010 & 0.0003 \\ & & \text { VH2SOP } & 0.0001 & 0.0009 & 0.0003\end{array}$

$\begin{array}{cccccc}5010 & 1000 & \text { H2S } & 0.0000 & 0.1087 & 0.0157 \\ & & \text { VH2SF } & 0.0000 & 0.0035 & 0.0005 \\ & \text { VH2SD } & 0.0008 & 0.0038 & 0.0017 \\ & \text { VH2SOP } & 0.0008 & 0.0036 & 0.0017\end{array}$

\begin{tabular}{cccccc}
5020 & 2000 & H2S & 0.0000 & 0.0384 & 0.0077 \\
& & VH2SF & 0.0000 & 0.0006 & 0.0001 \\
& & VH2SD & 0.0002 & 0.0007 & 0.0004 \\
& & VH2SOP & 0.0002 & 0.0007 & 0.0004 \\
5030 & \multirow{3}{*}{3000} & H2S & 0.0000 & 0.0396 & 0.0074 \\
& & VH2SF & 0.0000 & 0.0004 & 0.0001 \\
& & VH2SD & 0.0001 & 0.0004 & 0.0002 \\
& & VH2SOP & 0.0001 & 0.0004 & 0.0002
\end{tabular}


Tabela 1. Estimativa do coeficiente de herdabilidade (H2S) e variância do coeficiente de herdabilidade, estimada pelas fórmulas de Falconer (VH2SF), Dickerson (VH2SD) e Osborne \& Paterson, para diferentes coeficientes de herdabilidade esperados ( $\mathrm{H} 2)$.

\begin{tabular}{ccccccc}
\hline H2 & Caso & NObs & Estatistica & Minimo & Máximo & Média \\
\hline 0,2 & 1010 & 200 & H2S & 0.0000 & 0.3924 & 0.0446 \\
& & & VH2SF & 0.0000 & 0.0628 & 0.0071 \\
& & & VH2SD & 0.0016 & 0.0581 & 0.0107 \\
& & VH2SOP & 0.0016 & 0.0474 & 0.0101 \\
& & & & & \\
& \multirow{2}{*}{1020} & \multirow{2}{*}{400} & H2S & 0.0000 & 0.2005 & 0.0261 \\
& & VH2SF & 0.0000 & 0.0160 & 0.0021 \\
& & VH2SD & 0.0002 & 0.0155 & 0.0027 \\
& & VH2SOP & 0.0002 & 0.0140 & 0.0026
\end{tabular}

$\begin{array}{cccccc}1030 & 600 & \text { H2S } & 0.0000 & 0.1062 & 0.0154 \\ & & \text { VH2SF } & 0.0000 & 0.0057 & 0.0008 \\ & & \text { VH2SD } & 0.0001 & 0.0053 & 0.0011 \\ & & \text { VH2SOP } & 0.0001 & 0.0050 & 0.0011\end{array}$

$\begin{array}{cccccc}3010 & 600 & \mathrm{H} 2 \mathrm{~S} & 0.0000 & 0.0987 & 0.0275 \\ & & \text { VH2SF } & 0.0000 & 0.0053 & 0.0015 \\ & \text { VH2SD } & 0.0008 & 0.0053 & 0.0030 \\ & & \text { VH2SOP } & 0.0008 & 0.0051 & 0.0029\end{array}$

$\begin{array}{cccccc}3020 & 1200 & \text { H2S } & 0.0000 & 0.0507 & 0.0090 \\ & & \text { VH2SF } & 0.0000 & 0.0014 & 0.0002 \\ & \text { VH2SD } & 0.0002 & 0.0016 & 0.0007 \\ & & \text { VH2SOP } & 0.0002 & 0.0015 & 0.0007\end{array}$

$\begin{array}{cccccc}3030 & 1800 & \text { H2S } & 0.0000 & 0.0500 & 0.0066 \\ & & \text { VH2SF } & 0.0000 & 0.0009 & 0.0001 \\ & & \text { VH2SD } & 0.0001 & 0.0008 & 0.0003 \\ & & \text { VH2SOP } & 0.0001 & 0.0008 & 0.0003\end{array}$


Tabela 1. Estimativa do coeficiente de herdabilidade (H2S) e variância do coeficiente de herdabilidade, estimada pelas fórmulas de Falconer (VH2SF), Dickerson (VH2SD) e Osborne \& Paterson, para diferentes coeficientes de herdabilidade esperados $(\mathrm{H} 2)$.

H2 Caso NObs Estatística Minimo Máximo Média

\begin{tabular}{|c|c|c|c|c|c|c|}
\hline \multirow[t]{12}{*}{0,2} & \multirow[t]{4}{*}{5010} & \multirow[t]{4}{*}{1000} & $\mathrm{H} 2 \mathrm{~S}$ & 0.0000 & 0.1053 & 0.0128 \\
\hline & & & VH2SF & 0.0000 & 0.0034 & 0.0004 \\
\hline & & & $\mathrm{VH} 2 \mathrm{SD}$ & 0.0006 & 0.0040 & 0.0017 \\
\hline & & & VH2SOP & 0.0006 & 0.0038 & 0.0017 \\
\hline & \multirow[t]{4}{*}{5020} & \multirow[t]{4}{*}{2000} & $\mathrm{H} 2 \mathrm{~S}$ & 0.0000 & 0.0370 & 0.0078 \\
\hline & & & VH2SF & 0.0000 & 0.0006 & 0.0001 \\
\hline & & & VH2SD & 0.0002 & 0.0007 & 0.0004 \\
\hline & & & VH2SOP & 0.0002 & 0.0007 & 0.0004 \\
\hline & \multirow[t]{4}{*}{5030} & \multirow[t]{4}{*}{3000} & $\mathrm{H} 2 \mathrm{~S}$ & 0.0000 & 0.0419 & 0.0060 \\
\hline & & & VH2SF & 0.0000 & 0.0004 & 0.0001 \\
\hline & & & VH2SD & 0.0001 & 0.0005 & 0.0002 \\
\hline & & & VH2SOP & 0.0001 & 0.0004 & 0.0002 \\
\hline \multirow[t]{12}{*}{0,4} & \multirow[t]{4}{*}{1010} & \multirow[t]{4}{*}{200} & $\mathrm{H} 2 \mathrm{~S}$ & 0.0000 & 0.2402 & 0.0366 \\
\hline & & & $\mathrm{VH} 2 \mathrm{SF}$ & 0.0000 & 0.0384 & 0.0059 \\
\hline & & & VH2SD & 0.0015 & 0.0354 & 0.0089 \\
\hline & & & VH2SOP & 0.0015 & 0.0313 & 0.0085 \\
\hline & \multirow[t]{4}{*}{1020} & \multirow[t]{4}{*}{400} & $\mathrm{H} 2 \mathrm{~S}$ & 0.0000 & 0.1321 & 0.0170 \\
\hline & & & VH2SF & 0.0000 & 0.0106 & 0.0014 \\
\hline & & & VH2SD & 0.0002 & 0.0103 & 0.0021 \\
\hline & & & VH2SOP & 0.0002 & 0.0096 & 0.0021 \\
\hline & \multirow[t]{4}{*}{1030} & \multirow[t]{4}{*}{600} & $\mathrm{H} 2 \mathrm{~S}$ & 0.0000 & 0.1280 & 0.0157 \\
\hline & & & VH2SF & 0.0000 & 0.0068 & 0.0008 \\
\hline & & & VH2SD & 0.0001 & 0.0060 & 0.0011 \\
\hline & & & $\mathrm{VH} 2 \mathrm{SOP}$ & 0.0001 & 0.0056 & 0.0011 \\
\hline
\end{tabular}


Tabela 1. Estimativa do coeficiente de herdabilidade (H2S) e variância do coeficiente de herdabilidade, estimada pelas fórmulas de Falconer (VH2SF), Dickerson (VH2SD) e Osbome $\&$ Paterson, para diferentes coeficientes de herdabilidade esperados (H2).

\begin{tabular}{|c|c|c|c|c|c|c|}
\hline $\mathrm{H} 2$ & Caso & $\mathrm{NObs}$ & Estatistica & Minimo & Máximo & Média \\
\hline \multirow[t]{24}{*}{0,4} & 3010 & 600 & $\mathrm{H} 2 \mathrm{~S}$ & 0.0000 & 0.2018 & 0.0218 \\
\hline & & & VH2SF & 0.0000 & 0.0108 & 0.0012 \\
\hline & & & VH2SD & 0.0008 & 0.0102 & 0.0030 \\
\hline & & & VH2SOP & 0.0008 & 0.0092 & 0.0029 \\
\hline & 3020 & 1200 & $\mathrm{H} 2 \mathrm{~S}$ & 0.0000 & 0.0669 & 0.0090 \\
\hline & & & $\mathrm{VH} 2 \mathrm{SF}$ & 0.0000 & 0.0018 & 0.0002 \\
\hline & & & VH2SD & 0.0002 & 0.0018 & 0.0007 \\
\hline & & & VH2SOP & 0.0002 & 0.0018 & 0.0007 \\
\hline & 3030 & 1800 & $\mathrm{H} 2 \mathrm{~S}$ & 0.0000 & 0.0369 & 0.0069 \\
\hline & & & VH2SF & 0.0000 & 0.0007 & 0.0001 \\
\hline & & & VH2SD & 0.0001 & 0.0007 & 0.0003 \\
\hline & & & VH2SOP & 0.0001 & 0.0007 & 0.0003 \\
\hline & 5010 & 1000 & $\mathrm{H} 2 \mathrm{~S}$ & 0.0000 & 0.1270 & 0.0149 \\
\hline & & & $\mathrm{VH} 2 \mathrm{SF}$ & 0.0000 & 0.0041 & 0.0005 \\
\hline & & & VH2SD & 0.0008 & 0.0039 & 0.0017 \\
\hline & & & VH2SOP & 0.0008 & 0.0037 & 0.0017 \\
\hline & 5020 & 2000 & $\mathrm{H} 2 \mathrm{~S}$ & 0.0000 & 0.0556 & 0.0085 \\
\hline & & & VH2SF & 0.0000 & 0.0009 & 0.0001 \\
\hline & & & $\mathrm{VH} 2 \mathrm{SD}$ & 0.0002 & 0.0010 & 0.0005 \\
\hline & & & VH2SOP & 0.0002 & 0.0010 & 0.0005 \\
\hline & 5030 & 3000 & $\mathrm{H} 2 \mathrm{~S}$ & 0.0000 & 0.0347 & 0.0038 \\
\hline & & & $\mathrm{VH} 2 \mathrm{SF}$ & 0.0000 & 0.0004 & 0.0000 \\
\hline & & & VH2SD & 0.0001 & 0.0004 & 0.0002 \\
\hline & & & VH2SOP & 0.0001 & 0.0004 & 0.0002 \\
\hline
\end{tabular}


Tabela 1. Estimativa do coeficiente de herdabilidade (H2S) e variância do coeficiente de herdabilidade, estimada pelas formulas de Falconer (VH2SF), Dickerson (VH2SD) e Osborne $\varepsilon$ Paterson, para diferentes coeficientes de herdabilidade esperados (H2).

\begin{tabular}{|c|c|c|c|c|c|c|}
\hline $\mathrm{H} 2$ & Caso & NObs & Estatística & Minimo & Máximo & Média \\
\hline \multirow[t]{12}{*}{0,6} & 1010 & 200 & $\mathrm{H} 2 \mathrm{~S}$ & 0.0000 & 0.2299 & 0.0342 \\
\hline & & & VH2SF & 0.0000 & 0.0368 & 0.0055 \\
\hline & & & VH2SD & 0.0011 & 0.0295 & 0.0084 \\
\hline & & & VH2SOP & 0.0011 & 0.0270 & 0.0081 \\
\hline & 1020 & 400 & $\mathrm{H} 2 \mathrm{~S}$ & 0.0000 & 0.1122 & 0.0160 \\
\hline & & & $\mathrm{VH} 2 \mathrm{SF}$ & 0.0000 & 0.0090 & 0.0013 \\
\hline & & & VH2SD & 0.0002 & 0.0085 & 0.0020 \\
\hline & & & VH2SOP & 0.0002 & 0.0080 & 0.0019 \\
\hline & 1030 & 600 & $\mathrm{H} 2 \mathrm{~S}$ & 0.0000 & 0.1452 & 0.0150 \\
\hline & & & VH2SF & 0.0000 & 0.0077 & 0.0008 \\
\hline & & & $\mathrm{VH} 2 \mathrm{SD}$ & 0.0001 & 0.0073 & 0.0011 \\
\hline & & & VH2SOP & 0.0001 & 0.0067 & 0.0011 \\
\hline
\end{tabular}

$\begin{array}{cccccc}3010 & 600 & \text { H2S } & 0.0000 & 0.1292 & 0.0216 \\ & & \text { VH2SF } & 0.0000 & 0.0069 & 0.0012 \\ & & \text { VH2SD } & 0.0011 & 0.0067 & 0.0030 \\ & & \text { VH2SOP } & 0.0011 & 0.0063 & 0.0030\end{array}$

$\begin{array}{cccccc}3020 & 1200 & \text { H2S } & 0.0000 & 0.0979 & 0.0109 \\ & & \text { VH2SF } & 0.0000 & 0.0026 & 0.0003 \\ & & \text { VH2SD } & 0.0001 & 0.0026 & 0.0007 \\ & & \text { VH2SOP } & 0.0001 & 0.0025 & 0.0007\end{array}$

$\begin{array}{llclll}3030 & 1800 & \text { H2S } & 0.0000 & 0.0372 & 0.0058 \\ & & \text { VH2SF } & 0.0000 & 0.0007 & 0.0001 \\ & & \text { VH2SD } & 0.0001 & 0.0007 & 0.0003 \\ & & \text { VH2SOP } & 0.0001 & 0.0007 & 0.0003\end{array}$


Tabela 1. Estimativa do coeficiente de herdabilidade (H2S) e variância do coeficiente de herdabilidade, estimada pelas fórmulas de Falconer (VH2SF), Dickerson (VH2SD) e Osborne \& Paterson, para diferentes coeficientes de herdabilidade esperados (H2).

\begin{tabular}{ccccccc}
\hline H2 & Caso & N Obs & Estatistica & Mínimo & Máximo & Média \\
\hline 0,6 & 3030 & 1800 & H2S & 0.0000 & 0.0372 & 0.0058 \\
& & & VH2SF & 0.0000 & 0.0007 & 0.0001 \\
& & & VH2SD & 0.0001 & 0.0007 & 0.0003 \\
& & VH2SOP & 0.0001 & 0.0007 & 0.0003 \\
& & & & & \\
5 & 5010 & 1000 & H2S & 0.0000 & 0.1184 & 0.0141 \\
& & VH2SF & 0.0000 & 0.0038 & 0.0005 \\
& & VH2SD & 0.0005 & 0.0042 & 0.0017 \\
& & & VH2SOP & 0.0005 & 0.0039 & 0.0017
\end{tabular}

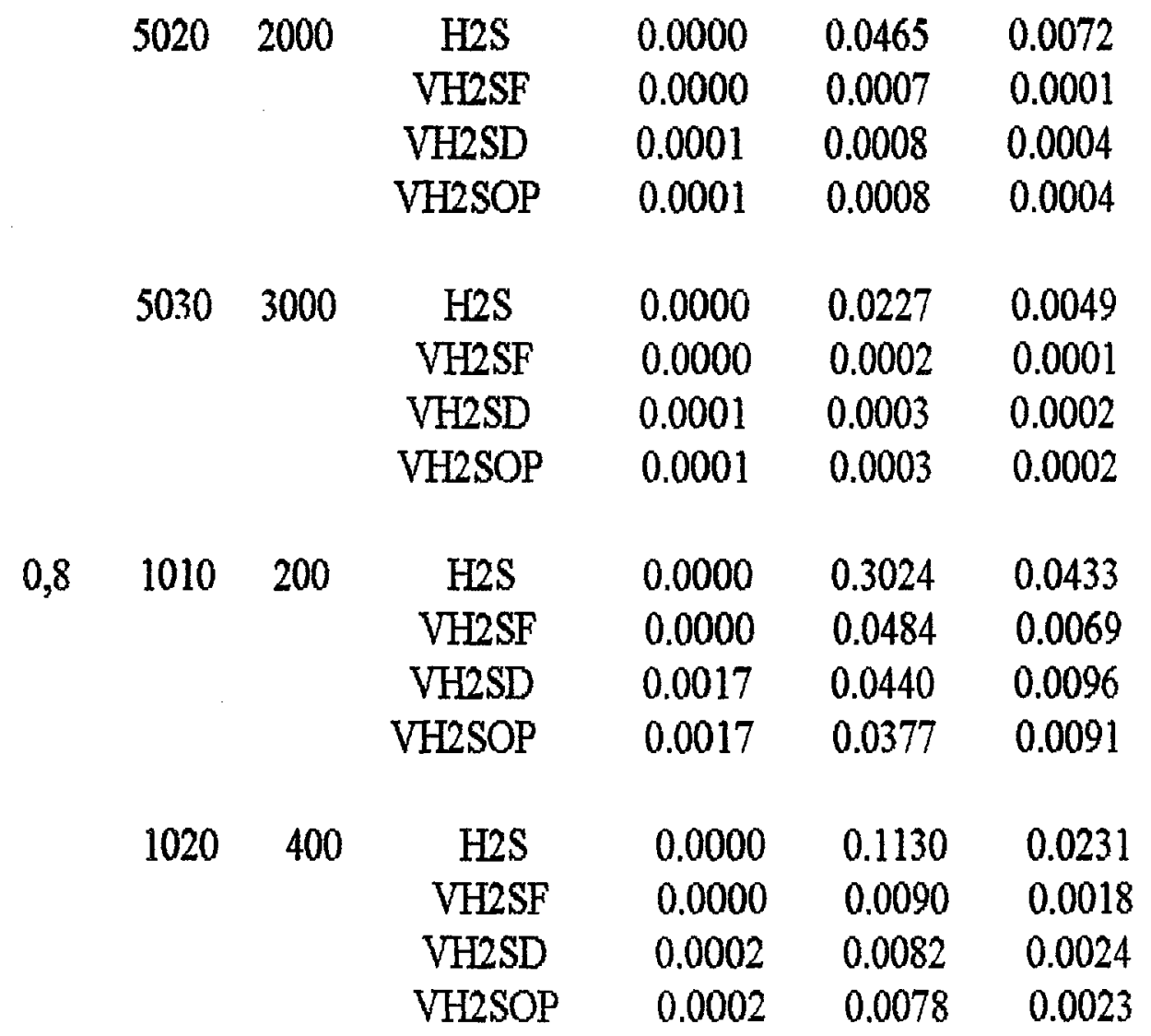


Tabela 1. Estimativa do coeficiente de herdabilidade (H2S) e variância do coeficiente de herdabilidade, estimada pelas formulas de Falconer (VH2SF), Dickerson (VH2SD) e Osborne $\&$ Paterson, para diferentes coeficientes de herdabilidade esperados $(\mathrm{H} 2)$.

H2 Caso NObs Estatistica Minimo Máximo Média

\begin{tabular}{|c|c|c|c|c|c|c|}
\hline \multirow{4}{*}{\multicolumn{2}{|c|}{1030}} & 600 & $\mathrm{H} 2 \mathrm{~S}$ & 0.0000 & 0.0632 & 0.0091 \\
\hline & & & VH2SF & 0.0000 & 0.0034 & 0.0005 \\
\hline & & & $\mathrm{VH} 2 \mathrm{SD}$ & 0.0001 & 0.0026 & 0.0009 \\
\hline & & & VH2SOP & 0.0001 & 0.0026 & 0.0009 \\
\hline \multirow{4}{*}{\multicolumn{2}{|c|}{3010}} & 600 & $\mathrm{H} 2 \mathrm{~S}$ & 0.0000 & 0.1361 & 0.0193 \\
\hline & & & VH2SF & 0.0000 & 0.0073 & 0.0010 \\
\hline & & & VH2SD & 0.0007 & 0.0073 & 0.0028 \\
\hline & & & VH2SOP & 0.0007 & 0.0069 & 0.0028 \\
\hline \multirow{4}{*}{\multicolumn{2}{|c|}{3020}} & 1200 & $\mathrm{H} 2 \mathrm{~S}$ & 0.0000 & 0.0691 & 0.0084 \\
\hline & & & $\mathrm{VH} 2 \mathrm{SF}$ & 0.0000 & 0.0018 & 0.0002 \\
\hline & & & VH2SD & 0.0002 & 0.0017 & 0.0007 \\
\hline & & & VH2SOP & 0.0002 & 0.0016 & 0.0007 \\
\hline \multirow{4}{*}{\multicolumn{2}{|c|}{3030}} & 1800 & $\mathrm{H} 2 \mathrm{~S}$ & 0.0000 & 0.0624 & 0.0071 \\
\hline & & & $\mathrm{VH} 2 \mathrm{SF}$ & 0.0000 & 0.0011 & 0.0001 \\
\hline & & & $\mathrm{VH} 2 \mathrm{SD}$ & 0.0001 & 0.0011 & 0.0003 \\
\hline & & & VH2SOP & 0.0001 & 0.0011 & 0.0003 \\
\hline \multirow{4}{*}{\multicolumn{2}{|c|}{5010}} & 1000 & $\mathrm{H} 2 \mathrm{~S}$ & 0.0000 & 0.1098 & 0.0102 \\
\hline & & & $\mathrm{VH} 2 \mathrm{SF}$ & 0.0000 & 0.0035 & 0.0003 \\
\hline & & & $\mathrm{VH} 2 \mathrm{SD}$ & 0.0007 & 0.0034 & 0.0015 \\
\hline & & & VH2SOP & 0.0007 & 0.0032 & 0.0015 \\
\hline \multirow{4}{*}{\multicolumn{2}{|c|}{5020}} & 2000 & $\mathrm{H} 2 \mathrm{~S}$ & 0.0000 & 0.0443 & 0.0072 \\
\hline & & & $\mathrm{VH} 2 \mathrm{SF}$ & 0.0000 & 0.0007 & 0.0001 \\
\hline & & & $\mathrm{VH} 2 \mathrm{SD}$ & 0.0002 & 0.0008 & 0.0004 \\
\hline & & & VH2SOP & 0.0002 & 0.0008 & 0.0004 \\
\hline
\end{tabular}


Tabela 1. Estimativa do coeficiente de herdabilidade (H2S) e variância do coeficiente de herdabilidade, estimada pelas formulas de Falconer (VH2SF), Dickerson (VH2SD) e Osbome $\varepsilon$ Paterson, para diferentes coeficientes de herdabilidade esperados ( $\mathrm{H} 2)$.

\begin{tabular}{lcccccc}
\hline H2 & Caso & NObs & Estatistica & Mínimo & Máximo & Média \\
\hline$j, 8$ & 5030 & \multirow{2}{*}{3000} & H2S & 0.0000 & 0.0366 & 0.0048 \\
& & & VH2SF & 0.0000 & 0.0004 & 0.0001 \\
& & & VH2SD & 0.0001 & 0.0004 & 0.0002 \\
& & & VH2SOP & 0.0001 & 0.0004 & 0.0002
\end{tabular}

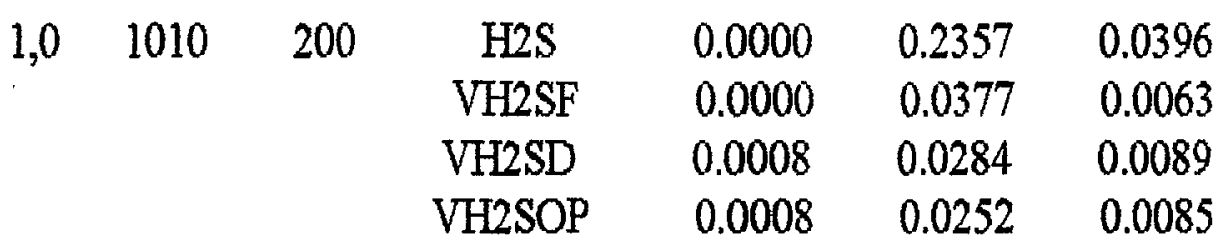

$\begin{array}{cccccc}1020 & 400 & \text { H2S } & 0.0000 & 0.1058 & 0.0152 \\ & & \text { VH2SF } & 0.0000 & 0.0085 & 0.0012 \\ & \text { VH2SD } & 0.0002 & 0.0065 & 0.0021 \\ & & \text { VH2SOP } & 0.0002 & 0.0061 & 0.0020\end{array}$

\begin{tabular}{|c|c|c|c|c|}
\hline 1030 & $\mathrm{H} 2 \mathrm{~S}$ & 0.0000 & 0.0540 & 0.0121 \\
\hline & VH2SF & 0.0000 & 0.0029 & 0.0006 \\
\hline & $\mathrm{VH} 2 \mathrm{SD}$ & 0.0001 & 0.0025 & 0.0010 \\
\hline & VH2SOP & 0.0001 & 0.0024 & 0.0010 \\
\hline
\end{tabular}

$\begin{array}{cccccc}3010 & 600 & \text { H2S } & 0.0000 & 0.1223 & 0.0270 \\ & & \text { VH2SF } & 0.0000 & 0.0065 & 0.0014 \\ & & \text { VH2SD } & 0.0005 & 0.0067 & 0.0030 \\ & & \text { VH2SOP } & 0.0005 & 0.0063 & 0.0030\end{array}$

$\begin{array}{cccccc}3020 & 1200 & \text { H2S } & 0.0000 & 0.0692 & 0.0142 \\ & & \text { VH2SF } & 0.0000 & 0.0018 & 0.0004 \\ & & \text { VH2SD } & 0.0002 & 0.0019 & 0.0007 \\ & & \text { VH2SOP } & 0.0002 & 0.0018 & 0.0007\end{array}$


Tabela 1. Estimativa do coeficiente de herdabilidade (H2S) e variância do coeficiente de herdabilidade, estimada pelas fórmulas de Falconer (VH2SF), Dickerson (VH2SD) e Osborne $\varepsilon$ Paterson, para diferentes coeficientes de herdabilidade esperados $(\mathrm{H} 2)$.

\begin{tabular}{|c|c|c|c|c|c|c|}
\hline $\mathrm{H} 2$ & Caso & NObs & Estatistica & Mínimo & Máximo & Média \\
\hline \multirow[t]{16}{*}{1,0} & \multirow[t]{4}{*}{3030} & \multirow[t]{4}{*}{1800} & $\mathrm{H} 2 \mathrm{~S}$ & 0.0000 & 0.0581 & 0.0071 \\
\hline & & & VH2SF & 0.0000 & 0.0010 & 0.0001 \\
\hline & & & VH2SD & 0.0001 & 0.0010 & 0.0003 \\
\hline & & & VH2SOP & 0.0001 & 0.0009 & 0.0003 \\
\hline & \multirow[t]{4}{*}{5010} & \multirow[t]{4}{*}{1000} & $\mathrm{H} 2 \mathrm{~S}$ & 0.0000 & 0.1247 & 0.0243 \\
\hline & & & VH2SF & 0.0000 & 0.0040 & 0.0008 \\
\hline & & & $\mathrm{VH} 2 \mathrm{SD}$ & 0.0005 & 0.0043 & 0.0019 \\
\hline & & & VH2SOP & 0.0005 & 0.0040 & 0.0019 \\
\hline & \multirow[t]{4}{*}{5020} & \multirow[t]{4}{*}{2000} & $\mathrm{H} 2 \mathrm{~S}$ & 0.0000 & 0.0599 & 0.0089 \\
\hline & & & $\mathrm{VH} 2 \mathrm{SF}$ & 0.0000 & 0.0010 & 0.0001 \\
\hline & & & VH2SD & 0.0001 & 0.0009 & 0.0004 \\
\hline & & & VH2SOP & 0.0001 & 0.0009 & 0.0004 \\
\hline & \multirow[t]{4}{*}{5030} & \multirow[t]{4}{*}{3000} & $\mathrm{H} 2 \mathrm{~S}$ & 0.0000 & 0.0251 & 0.0048 \\
\hline & & & $\mathrm{VH} 2 \mathrm{SF}$ & 0.0000 & 0.0003 & 0.0001 \\
\hline & & & $\mathrm{VH} 2 \mathrm{SD}$ & 0.0001 & 0.0003 & 0.0002 \\
\hline & & & VH2SOP & 0.0001 & 0.0003 & 0.0002 \\
\hline
\end{tabular}

A tabela 1, mostra que, para a estatística $\mathrm{H} 2 \mathrm{~S}$, estimativa do coeficiente de herdabilidade, o valor minimo é sempre zero. Isto acontece, devido à ocorrência de estimativas negativas dos componentes de variância, as quais foram admitidas como nulas. Como consequência, tem-se altas frequências de se obter estimativas negativas do coeficiente de herdabilidade, (Apêndice 1). 
O valor minimo para a estatistica $\mathrm{VH} 2 \mathrm{SF}$, variância estimada pela formula de Falconer, foi sempre zero. Isto ocorre devido ao emprego da fómula, que é função apenas do coeficiente de herdabilidade.

Para a estatistica $\mathrm{H} 2 \mathrm{~S}$, coeficiente de herdabilidade estimado, pode-se observar valores máximos maiores que 1. Este fato deve-se ao intervalo de variação desta variável, conforme pode-se observar em SINGH (1993).

Para as estatisticas VH2SD, variância estimada pela fórmula de Dickerson e VH2SOP, variância estimada pela fórmula de Osborne e Paterson, adaptada por Barbin, os valores minimos praticamente não diferem. Na maioria dos casos, os máximos e as médias foram menores, quando empregou-se a fórmula de Osborne e Paterson, adaptada por Barbin. Para grandes amostras, os valores minimos, máximos e as médias praticamente não diferem. 


\section{CONCLUSÕES}

Neste trabalho, podemos concluir, de uma maneira geral, que:

5.1. O emprego do método dos.momentos para estimar os componentes de variância do modelo e, consequentemente, a estimativa do coeficiente de herdabilidade, apresenta uma desvantagem, devido às altas frequências de ocorrência de estimativas negativas do coeficiente herdabilidade, o que confirma GLL $\varepsilon$ JENSEN (1968).

5.2. Quando se emprega o método dos momentos para estimar o coeficiente de herdabilidade e admiti-se as estimativas dos componentes de variância do modelo como nulas, a fórmula proposta por FALCONER (1960), mostra-se ineficiente devido às elevadas frequências de ocorrência de estimativas negativas do coeficiente herdabilidade.

5.3. Para pequenas amostras, as fórmulas de DICKERSON (1969) e OSBORNE \& PATERSON (1952), adaptada por BARBIN (1969), para estimar a variância do coeficiente de herdabilidade, claramente diferem entre si. Entretanto, a fórmula de OSBORNE \& PATERSON, adaptada por BARBIN (1969), sempre apresentou menor variância dentre todas as situações simuladas. Mas, à medida que aumenta-se o tamanho da amostra, quer seja pelo aumento do numero de machos ou 
fềmeas, as variâncias tendem a tornarem-se iguais, ou mesmo igual para grandes amostras. 


\section{REFERÊNCIAS BIBLIOGRÁFICAS}

BARBIN, D. A herdabilidade do peso aos dezoito meses do gado Canchin. Piracicaba,

1969. 67p. (Doutorado - Escola Superior de Agricultura "Luiz de Queiroz" / USP).

BOX, G. E. \& MULLER, M. E. A note on the geration of random normal deviates. Annals of Mathematical Statistics, Ann Arbor, 29: 610 - 11, 1958.

BROEMELING, L. D. Confidence intervals for variance measure of heritability. Biometrics, Washington, 25: $424-27,1969$.

DICKERSON, G. E. Techniques for research in quantitative animal genetics. In: techniques and procedures in animal production research. American Society of Animal Science, New York. 1969. 228p.

FALCONER, D. S. Introduction to quantitative genetics. Edinburgh, Oliver and Boyd, 1960. 365p.

FISHER, R. A. Statistical methods for research workers. Edinburgh, Oliver and Boyd, 1954. 362p.

GLL, J. L. $\varepsilon$ JENSEN, E. L. Probability of obtaining negative estimates of heritability. Biometrics, Washington, 24: $517-26,1968$.

GRAYBLL, F. A.; MARTIN, F.; GODFREY, G. Confidence intervals for variance ratios specifying genetic heritability. Biometrics, Washington, 12: $99-109$, 1956.

HILL, B. M. Inference about variance components in the one-way model. Journal of American Statistical Association, Washington, 60: 806-25, 1965. 
KEMPTHORNE, O. An introduction to genetic statistics. Iowa, Iowa State University Press, 1957. 545p.

KEMPTHORNE, O. Comments on Searle's paper. Biometrics, Washington, 40: $782-84,1968$

KIILAM, B. An overview of the SAS system random number generators. In: ANNUAL SAS USERS GROUP INTERNATIONAL CONFERENCE, 12. Dallas, 1987. Proceedings. Cary, SAS INSTTTUTE, 1987. p. 1059-64. NELDER, J. A. The interpretation of negative components of variance. Biometrika, Londres, 41: 544 - 48, 1954.

OSBORNE, R. \& PATERSON, W. S. B. On the sampling variance of heritability estimates derived from variance analysis. Proceedings of the Royal Society, Edinburgh, 64: 456-61, 1952.

ROBERTSON, A. Experimental design in the evaluation of genetic parameters. Biometrics, Washington, 15: $219-226,1959$.

SILVA, R. G. Métodos de genética quantitativa aplicados ao melhoramento animal. Ribeirão Preto, Sociedade Brasileira de Genética, 1982. 162p.

SINGH, B. On the estimation of heritability from unbalanced data. Biometrical Journal, Berlin, 35: 823-31, 1993.

SWINGER, L. A.; HARVEY, W. R.; EVERSON, D. O.; GREGORY, K. E. The variance of intraclass correlation involving groups with one observation. Biometrics, Washington, 20: $818-26,1964$.

VANVLECK, L. D. \& HENDERSON, C. R. Empirical sampling estimates of genetic correlations. Biometrics, Washington, 17: $359-371,1961$. 
A P E N D I C E 1 
Tabela 2. Frequências de ocorrências de estimativas negativas (0) e positivas (1) do coeficiente de herdabilidade (H2) para vários casos de combinações de machos e fềmeas, com dois descendentes por acasalmento e diferentes coeficientes de herdabilidade esperados $\left(\mathrm{H}^{2}\right)$.

\begin{tabular}{|c|c|c|c|c|}
\hline $\mathrm{H}^{2}$ & Caso & $\mathrm{H} 2$ & Freq & $\%$ \\
\hline \multirow[t]{26}{*}{0,0} & 22 & 0 & 28 & 56.0 \\
\hline & & 1 & 22 & 44.0 \\
\hline & 24 & 0 & 28 & 56.0 \\
\hline & & 1 & 22 & 44.0 \\
\hline & 26 & 0 & 27 & 54.0 \\
\hline & & 1 & 23 & 46.0 \\
\hline & 28 & 0 & 24 & 48.0 \\
\hline & & 1 & 26 & 52.0 \\
\hline & 42 & 0 & 26 & 52.0 \\
\hline & & 1 & 24 & 48.0 \\
\hline & 44 & 0 & 27 & 54.0 \\
\hline & & 1 & 23 & 46.0 \\
\hline & 46 & 0 & 26 & 52.0 \\
\hline & & 1 & 24 & 48.0 \\
\hline & 48 & 0 & 30 & 60.0 \\
\hline & & 1 & 20 & 40.0 \\
\hline & 62 & 0 & 24 & 48.0 \\
\hline & & 1 & 26 & 52.0 \\
\hline & 64 & 0 & 31 & 62.0 \\
\hline & & 1 & 19 & 38.0 \\
\hline & 66 & 0 & 28 & 56.0 \\
\hline & & 1 & 22 & 44.0 \\
\hline & 68 & 0 & 36 & 72.0 \\
\hline & & 1 & 14 & 28.0 \\
\hline & 82 & 0 & 26 & 52.0 \\
\hline & & 1 & 24 & 48.0 \\
\hline
\end{tabular}


Tabela 2. Frequências de ocorrências de estimativas negativas (0) e positivas (1) do coeficiente de herdabilidade (H2) para vários casos de combinações de machos e fểmeas, com dois descendentes por acasalmento e diferentes coeficientes de herdabilidade esperados $\left(\mathrm{H}^{2}\right)$.

\begin{tabular}{ccccc}
\hline $\mathrm{H}^{2}$ & Caso & $\mathrm{H} 2$ & Freq & $\%$ \\
\hline 0,0 & 84 & 0 & 24 & 48.0 \\
& & 1 & 26 & 52.0
\end{tabular}

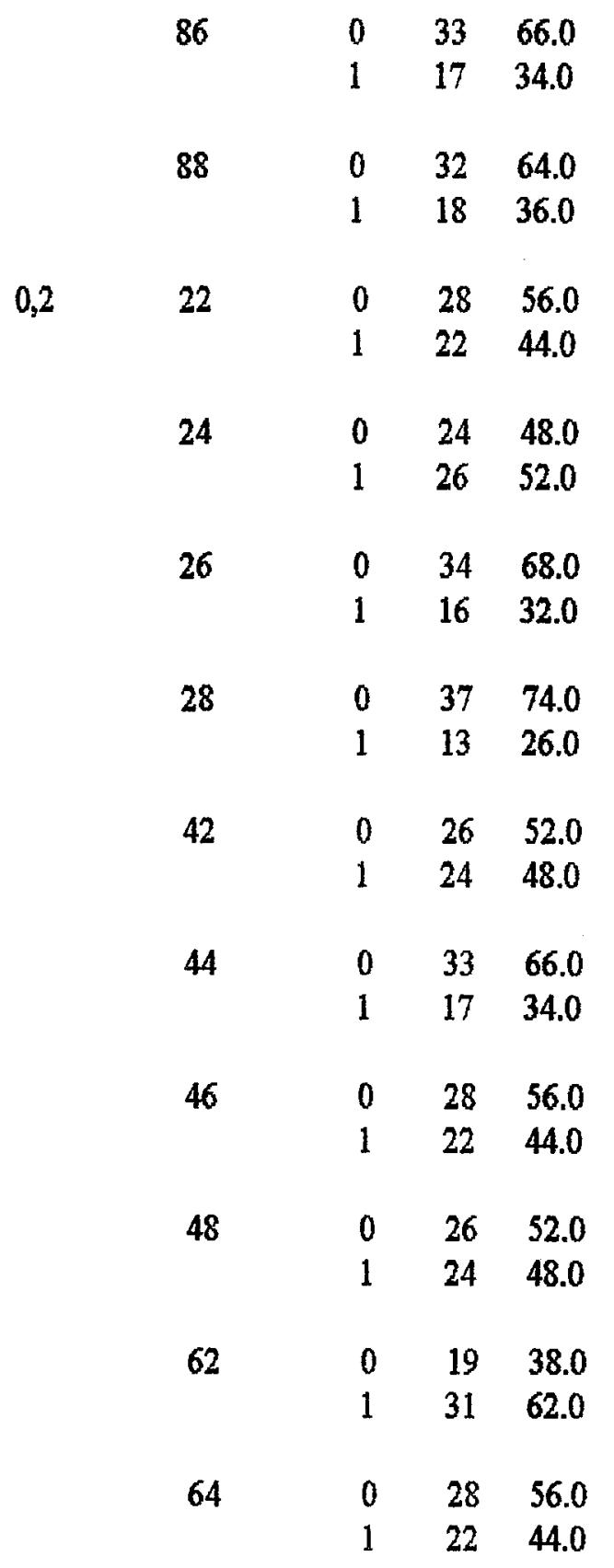


Tabela 2. Frequências de ocorrências de estimativas negativas (0) e positivas (1) do coeficiente de herdabilidade (H2) para vários casos de combinaçóes de machos e fềmeas, com dois descendentes por acasalmento e diferentes coeficientes de herdabilidade esperados $\left(\mathrm{H}^{2}\right)$.

\begin{tabular}{|c|c|c|c|c|}
\hline $\mathrm{H}^{2}$ & Caso & $\mathrm{H} 2$ & Freq & $\%$ \\
\hline & \multirow[t]{2}{*}{66} & 0 & 29 & 58.0 \\
\hline & & 1 & 21 & 42.0 \\
\hline & \multirow[t]{2}{*}{68} & 0 & 26 & 52.0 \\
\hline & & 1 & 24 & 48.0 \\
\hline & \multirow[t]{2}{*}{82} & 0 & 24 & 48.0 \\
\hline & & 1 & 26 & 52.0 \\
\hline & \multirow[t]{2}{*}{84} & 0 & 27 & 54.0 \\
\hline & & 1 & 23 & 46.0 \\
\hline & \multirow[t]{2}{*}{86} & 0 & 32 & 64.0 \\
\hline & & 1 & 18 & 36.0 \\
\hline & \multirow[t]{2}{*}{88} & 0 & 27 & 54.0 \\
\hline & & 1 & 23 & 46.0 \\
\hline \multirow[t]{14}{*}{0,4} & \multirow[t]{2}{*}{22} & 0 & 28 & 56.0 \\
\hline & & 1 & 22 & 44.0 \\
\hline & \multirow[t]{2}{*}{24} & 0 & 28 & 56.0 \\
\hline & & 1 & 22 & 44.0 \\
\hline & \multirow[t]{2}{*}{26} & 0 & 30 & 60.0 \\
\hline & & 1 & 20 & 40.0 \\
\hline & \multirow[t]{2}{*}{28} & 0 & 36 & 72.0 \\
\hline & & 1 & 14 & 28.0 \\
\hline & \multirow[t]{2}{*}{42} & 0 & 27 & 54.0 \\
\hline & & 1 & 23 & 46.0 \\
\hline & \multirow[t]{2}{*}{44} & 0 & 28 & 56.0 \\
\hline & & 1 & 22 & 44.0 \\
\hline & \multirow[t]{2}{*}{46} & 0 & 26 & 52.0 \\
\hline & & 1 & 24 & 48.0 \\
\hline
\end{tabular}


Tabela 2. Frequências de ocorrências de estimativas negativas ( 0 ) e positivas (1) do coeficiente de herdabilidade $(\mathrm{H} 2)$ para varios casos de combinaçôes de machos e fềmeas, com dois descendentes por acasalmento e diferentes coeficientes de herdabilidade esperados $\left(\mathrm{H}^{2}\right)$.

\begin{tabular}{|c|c|c|c|c|}
\hline $\mathrm{H}^{2}$ & Caso & $\mathrm{H} 2$ & Freq & $\%$ \\
\hline \multirow[t]{18}{*}{0,4} & 48 & 0 & 24 & 48.0 \\
\hline & & 1 & 26 & \\
\hline & 62 & 0 & 30 & 60.0 \\
\hline & & 1 & 20 & 40.0 \\
\hline & 64 & 0 & 27 & 54.0 \\
\hline & & 1 & 23 & 46.0 \\
\hline & 66 & 0 & 33 & 66,0 \\
\hline & & 1 & 17 & 34.0 \\
\hline & 68 & 0 & 29 & 58.0 \\
\hline & & 1 & 21 & 42.0 \\
\hline & 82 & 0 & 26 & 52.0 \\
\hline & & 1 & 24 & 48.0 \\
\hline & 84 & 0 & 25 & 50.0 \\
\hline & & 1 & 25 & 50.0 \\
\hline & 86 & 0 & 26 & 52.0 \\
\hline & & 1 & 24 & 48.0 \\
\hline & 88 & 0 & 30 & 60.0 \\
\hline & & 1 & 20 & 40.0 \\
\hline \multirow[t]{8}{*}{0,6} & 22 & 0 & 27 & 54.0 \\
\hline & & 1 & 23 & 46.0 \\
\hline & 24 & 0 & 31 & 62.0 \\
\hline & & 1 & 19 & 38.0 \\
\hline & 26 & 0 & 35 & 70.0 \\
\hline & & 1 & 15 & 30.0 \\
\hline & 28 & 0 & 38 & 76.0 \\
\hline & & 1 & 12 & 24.0 \\
\hline
\end{tabular}


Tabela 2. Frequências de ocorrências de estimativas negativas ( 0 ) e positivas (1) do coeficiente de herdabilidade (H2) para vários casos de combinações de machos e fềmeas, com dois descendentes por acasalmento e diferentes coeficientes de herdabilidade esperados $\left(\mathrm{H}^{2}\right)$.

\begin{tabular}{|c|c|c|c|c|}
\hline $\mathrm{H}^{2}$ & Caso & $\mathrm{H} 2$ & Freq & $\%$ \\
\hline & \multirow[t]{2}{*}{42} & 0 & 26 & 52.0 \\
\hline & & 1 & 24 & 48.0 \\
\hline & \multirow[t]{2}{*}{44} & 0 & 32 & 64.0 \\
\hline & & 1 & 18 & 36.0 \\
\hline & \multirow[t]{2}{*}{46} & 0 & 29 & 58.0 \\
\hline & & 1 & 21 & 42.0 \\
\hline & \multirow[t]{2}{*}{48} & 0 & 29 & 58.0 \\
\hline & & 1 & 21 & 42.0 \\
\hline & \multirow[t]{2}{*}{62} & 0 & 30 & 60.0 \\
\hline & & 1 & 20 & 40.0 \\
\hline & \multirow[t]{2}{*}{64} & 0 & 24 & 48.0 \\
\hline & & 1 & 26 & 52.0 \\
\hline & \multirow[t]{2}{*}{66} & 0 & 28 & 56.0 \\
\hline & & 1 & 22 & 44.0 \\
\hline & \multirow[t]{2}{*}{68} & 0 & 27 & 54.0 \\
\hline & & 1 & 23 & 46.0 \\
\hline & \multirow[t]{2}{*}{82} & 0 & 20 & 40.0 \\
\hline & & 1 & 30 & 60.0 \\
\hline & \multirow[t]{2}{*}{84} & 0 & 29 & 58.0 \\
\hline & & 1 & 21 & 42.0 \\
\hline & \multirow[t]{2}{*}{86} & 0 & 33 & 66.0 \\
\hline & & 1 & 17 & 34.0 \\
\hline & \multirow[t]{2}{*}{88} & 0 & 24 & 48.0 \\
\hline & & 1 & 26 & 52.0 \\
\hline \multirow[t]{2}{*}{0,8} & \multirow[t]{2}{*}{22} & 0 & 31 & 62.0 \\
\hline & & 1 & 19 & 38.0 \\
\hline
\end{tabular}


Tabela 2. Frequências de ocorrências de estimativas negativas ( 0 ) e positivas (1) do coeficiente de herdabilidade (H2) para vários casos de combinações de machos e fềmeas, com dois descendentes por acasalmento e diferentes coeficientes de herdabilidade esperados $\left(\mathrm{H}^{2}\right)$.

\begin{tabular}{|c|c|c|c|c|}
\hline $\mathrm{H}^{2}$ & Caso & $\mathrm{H} 2$ & Freq & $\%$ \\
\hline \multirow[t]{26}{*}{0,8} & 24 & 0 & 32 & 64.0 \\
\hline & & 1 & 18 & 36.0 \\
\hline & 26 & 0 & 28 & 56.0 \\
\hline & & 1 & 22 & 44.0 \\
\hline & 28 & 0 & 32 & 64.0 \\
\hline & & 1 & 18 & 36.0 \\
\hline & 42 & 0 & 19 & 38.0 \\
\hline & & 1 & 31 & 62.0 \\
\hline & 44 & 0 & 34 & 68.0 \\
\hline & & 1 & 16 & 32.0 \\
\hline & 46 & 0 & 24 & 48.0 \\
\hline & & 1 & 26 & 52.0 \\
\hline & 48 & 0 & 25 & 50.0 \\
\hline & & 1 & 25 & 50.0 \\
\hline & 62 & 0 & 29 & 58.0 \\
\hline & & 1 & 21 & 42.0 \\
\hline & 64 & 0 & 28 & 56.0 \\
\hline & & 1 & 22 & 44.0 \\
\hline & 66 & 0 & 31 & 62.0 \\
\hline & & 1 & 19 & 38.0 \\
\hline & 68 & 0 & 27 & 54.0 \\
\hline & & 1 & 23 & 46.0 \\
\hline & 82 & 0 & 26 & 52.0 \\
\hline & & 1 & 24 & 48.0 \\
\hline & 84 & 0 & 28 & 56.0 \\
\hline & & 1 & 22 & 44.0 \\
\hline
\end{tabular}


Tabela 2. Frequências de ocorrências de estimativas negativas (0) e positivas (1) do coeficiente de herdabilidade ( $\mathrm{H} 2$ ) para vários casos de combinaçôes de machos e fềmeas, com dois descendentes por acasalmento e diferentes coeficientes de herdabilidade esperados $\left(\mathrm{H}^{2}\right)$.

\begin{tabular}{lcccc}
\hline $\mathrm{H}^{2}$ & Caso & $\mathrm{H} 2$ & Freq & $\%$ \\
\hline 0,8 & 86 & 0 & 27 & 54.0 \\
& & 1 & 23 & 46.0
\end{tabular}

$\begin{array}{llll}88 & 0 & 24 & 48.0\end{array}$

$1 \quad 26 \quad 52.0$

1,0

\begin{tabular}{|c|c|c|c|}
\hline 22 & 0 & 32 & 64.0 \\
\hline & 1 & 18 & 36.0 \\
\hline 24 & 0 & 37 & 74.0 \\
\hline & 1 & 13 & 26.0 \\
\hline 26 & 0 & 33 & 66. \\
\hline & 1 & 17 & 34.0 \\
\hline
\end{tabular}

28

$\begin{array}{lll}0 & 30 & 60.0 \\ 1 & 20 & 40.0\end{array}$

42

$\begin{array}{lll}0 & 30 & 60.0 \\ 1 & 20 & 40.0\end{array}$

44

$\begin{array}{lll}0 & 22 & 44.0\end{array}$

$128 \quad 56.0$

$\begin{array}{llll}46 & 0 & 25 & 50.0\end{array}$

$\begin{array}{lll}1 & 25 & 50.0\end{array}$

48

$\begin{array}{lll}0 & 28 & 56.0\end{array}$

$1 \quad 22 \quad 44.0$

62

$\begin{array}{lll}0 & 23 & 46.0\end{array}$

$\begin{array}{lll}1 & 27 & 54.0\end{array}$

64

$\begin{array}{lll}0 & 27 & 54.0\end{array}$

$123 \quad 46.0$

$\begin{array}{llll}66 & 0 & 33 & 66.0\end{array}$

$1 \quad 17 \quad 34.0$

(continua) 
Tabela 2. Frequências de ocorrências de estimativas negativas ( 0 e positivas (1) do coeficiente de herdabilidade $(\mathrm{H} 2)$ para vários casos de combinações de machos e fêmeas, com dois descendentes por acasaimento e diferentes coeficientes de herdabilidade esperados $\left(\mathrm{H}^{2}\right)$.

\begin{tabular}{|c|c|c|c|c|}
\hline $\mathrm{H}^{2}$ & Caso & $\mathrm{H} 2$ & Freq & $\%$ \\
\hline \multirow[t]{9}{*}{1,0} & 68 & $\begin{array}{l}0 \\
1\end{array}$ & $\begin{array}{l}25 \\
25\end{array}$ & $\begin{array}{l}50.0 \\
50.0\end{array}$ \\
\hline & 82 & 0 & 26 & 52.0 \\
\hline & & 1 & 24 & 48.0 \\
\hline & 84 & 0 & 28 & 56.0 \\
\hline & & 1 & 22 & 44.0 \\
\hline & 86 & 0 & 24 & 48.0 \\
\hline & & 1 & 26 & 52.0 \\
\hline & 88 & 0 & 31 & 62.0 \\
\hline & & 1 & 19 & 38.0 \\
\hline \multirow[t]{16}{*}{0,0} & 1010 & 0 & 30 & 60.0 \\
\hline & & 1 & 20 & 40.0 \\
\hline & 1020 & 0 & 25 & 50.0 \\
\hline & & 1 & 25 & 50.0 \\
\hline & 1030 & 0 & 28 & 56.0 \\
\hline & & 1 & 22 & 44.0 \\
\hline & 3010 & 0 & 29 & 58.0 \\
\hline & & 1 & 21 & 42.0 \\
\hline & 3020 & 0 & 30 & 60.0 \\
\hline & & 1 & 20 & 40.0 \\
\hline & 3030 & 0 & 31 & 62.0 \\
\hline & & 1 & 19 & 38.0 \\
\hline & 5010 & 0 & 28 & 56.0 \\
\hline & & 1 & 22 & 44.0 \\
\hline & 5020 & 0 & 29 & 58.0 \\
\hline & & 1 & 21 & 42.0 \\
\hline
\end{tabular}


Tabela 2. Frequências de ocorrências de estimativas negativas (0) e positivas (1) do coeficiente de herdabilidade (H2) para vários casos de combinações de machos e fềmeas, com dois descendentes por acasalmento e diferentes coeficientes de herdabilidade esperados $\left(\mathrm{H}^{2}\right)$.

\begin{tabular}{|c|c|c|c|c|}
\hline $\mathrm{H}^{2}$ & Caso & $\mathrm{H} 2$ & Freq & $\%$ \\
\hline \multirow[t]{2}{*}{0,0} & 5030 & 0 & 21 & 42.0 \\
\hline & & 1 & 29 & 58.0 \\
\hline \multirow[t]{18}{*}{0,2} & 1010 & 0 & 20 & 40.0 \\
\hline & & 1 & 30 & 60.0 \\
\hline & 1020 & 0 & 27 & 54.0 \\
\hline & & 1 & 23 & 46.0 \\
\hline & 1030 & 0 & 25 & 50.0 \\
\hline & & 1 & 25 & 50.0 \\
\hline & 3010 & 0 & 23 & 46.0 \\
\hline & & 1 & 27 & 54.0 \\
\hline & 3020 & 0 & 25 & 50.0 \\
\hline & & 1 & 25 & 50.0 \\
\hline & 3030 & 0 & 30 & 60.0 \\
\hline & & 1 & 20 & 40.0 \\
\hline & 5010 & 0 & 22 & 44.0 \\
\hline & & 1 & 28 & 56.0 \\
\hline & 5020 & 0 & 26 & 52.0 \\
\hline & & 1 & 24 & 48.0 \\
\hline & 5030 & 0 & 27 & 54.0 \\
\hline & & 1 & 23 & 46.0 \\
\hline \multirow[t]{6}{*}{0,4} & 1010 & 0 & 23 & 46.0 \\
\hline & & 1 & 27 & 54.0 \\
\hline & 1020 & 0 & 29 & 58.0 \\
\hline & & 1 & 21 & 42.0 \\
\hline & 1030 & 0 & 30 & 60.0 \\
\hline & & 1 & 20 & 40.0 \\
\hline
\end{tabular}


Tabela 2. Frequências de ocorrências de estimativas negativas (0) e positivas (1) do coeficiente de herdabilidade (H2) para vários casos de combinaçóes de machos e fềmeas, com dois descendentes por acasalmento e diferentes coeficientes de herdabilidade esperados $\left(\mathrm{H}^{2}\right)$.

\begin{tabular}{|c|c|c|c|c|}
\hline $\mathrm{H}^{2}$ & Caso & $\mathrm{H} 2$ & Freq & $\%$ \\
\hline \multirow[t]{12}{*}{0,4} & 3010 & 0 & 27 & 54.0 \\
\hline & & 1 & 23 & 46.0 \\
\hline & 3020 & 0 & 28 & 56.0 \\
\hline & & 1 & 22 & 44.0 \\
\hline & 3030 & 0 & 23 & 46.0 \\
\hline & & 1 & 27 & 54.0 \\
\hline & 5010 & 0 & 26 & 52.0 \\
\hline & & 1 & 24 & 48.0 \\
\hline & 5020 & 0 & 18 & 36.0 \\
\hline & & 1 & 32 & 64.0 \\
\hline & 5030 & 0 & 36 & 72.0 \\
\hline & & 1 & 14 & 28.0 \\
\hline \multirow[t]{14}{*}{0,6} & 1010 & 0 & 29 & 58.0 \\
\hline & & 1 & 21 & 42.0 \\
\hline & 1020 & 0 & 34 & 68.0 \\
\hline & & 1 & 16 & 32.0 \\
\hline & 1030 & 0 & 24 & 48.0 \\
\hline & & 1 & 26 & 52.0 \\
\hline & 3010 & 0 & 26 & 52.0 \\
\hline & & 1 & 24 & 48.0 \\
\hline & 3020 & 0 & 25 & 50.0 \\
\hline & & 1 & 25 & 50.0 \\
\hline & 3030 & 0 & 27 & 54.0 \\
\hline & & 1 & 23 & 46.0 \\
\hline & 5010 & 0 & 28 & 56.0 \\
\hline & & 1 & 22 & 44.0 \\
\hline
\end{tabular}


Tabela 2. Frequências de ocorrências de estimativas negativas $(0)$ e positivas (1) do coeficiente de herdabilidade (H2) para vários casos de combinaçőes de machos e fềmeas, com dois descendentes por acasalmento e diferentes coeficientes de herdabilidade esperados $\left(\mathrm{H}^{2}\right)$.

\begin{tabular}{|c|c|c|c|c|}
\hline $\mathrm{H}^{2}$ & Caso & $\mathrm{H}_{2}$ & Freq & $\%$ \\
\hline \multirow[t]{4}{*}{0,6} & 5020 & 0 & 29 & 58.0 \\
\hline & & 1 & 21 & 42.0 \\
\hline & 5030 & 0 & 24 & 48.0 \\
\hline & & 1 & 26 & 52.0 \\
\hline \multirow[t]{18}{*}{0,8} & 1010 & 0 & 24 & 48.0 \\
\hline & & 1 & 26 & 52.0 \\
\hline & 1020 & 0 & 23 & 46.0 \\
\hline & & 1 & 27 & 54.0 \\
\hline & 1030 & 0 & 28 & 56.0 \\
\hline & & 1 & 22 & 44.0 \\
\hline & 3010 & 0 & 28 & 56.0 \\
\hline & & 1 & 22 & 44.0 \\
\hline & 3020 & 0 & 28 & 56.0 \\
\hline & & 1 & 22 & 44.0 \\
\hline & 3030 & 0 & 28 & 56.0 \\
\hline & & 1 & 22 & 44.0 \\
\hline & 5010 & 0 & 36 & 72.0 \\
\hline & & 1 & 14 & 28.0 \\
\hline & 5020 & 0 & 26 & 52.0 \\
\hline & & 1 & 24 & 48.0 \\
\hline & 5030 & 0 & 30 & 60.0 \\
\hline & & 1 & 20 & 40.0 \\
\hline \multirow[t]{4}{*}{1,0} & 1010 & 0 & 28 & 56.0 \\
\hline & & 1 & 22 & 44.0 \\
\hline & 1020 & 0 & 27 & 54.0 \\
\hline & & 1 & 23 & 46.0 \\
\hline
\end{tabular}


Tabela 2. Frequências de ocorrências de estimativas negativas ( 0 ) e positivas (1) do coeficiente de herdabilidade (H2) para vários casos de combinaçốes de machos e fềmeas, com dois descendentes por acasalmento e diferentes coeficientes de herdabilidade esperados $\left(\mathrm{H}^{2}\right)$.

\begin{tabular}{|c|c|c|c|c|}
\hline $\mathrm{H}^{2}$ & Caso & $\mathrm{H} 2$ & Freq & $\%$ \\
\hline \multirow[t]{14}{*}{1,0} & 1030 & 0 & 21 & 42.0 \\
\hline & & 1 & 29 & 58.0 \\
\hline & 3010 & 0 & 24 & 48.0 \\
\hline & & 1 & 26 & 52.0 \\
\hline & 3020 & 0 & 29 & 58.0 \\
\hline & & 1 & 21 & 42.0 \\
\hline & 3030 & 0 & 26 & 52.0 \\
\hline & & 1 & 24 & 48.0 \\
\hline & 5010 & 0 & 21 & 42.0 \\
\hline & & 1 & 29 & 58.0 \\
\hline & 5020 & 0 & 29 & 58.0 \\
\hline & & 1 & 21 & 42.0 \\
\hline & 5030 & 0 & 24 & 48.0 \\
\hline & & 1 & 26 & 52.0 \\
\hline
\end{tabular}




\section{APENDICE 2}


Anexo 1. Programa computacional empregado na simulação dos dados, desenvolvido em lin guagem "SAS", versão 6.04 para microcomputadores compativeis com a linha IBM - PC.

options ps $=500$ nodate $\mathrm{ls}=70$;

data saidal;

/*faz a simulação dos dados*/ do $\mathrm{I}=10$ to 50 by 20 ;

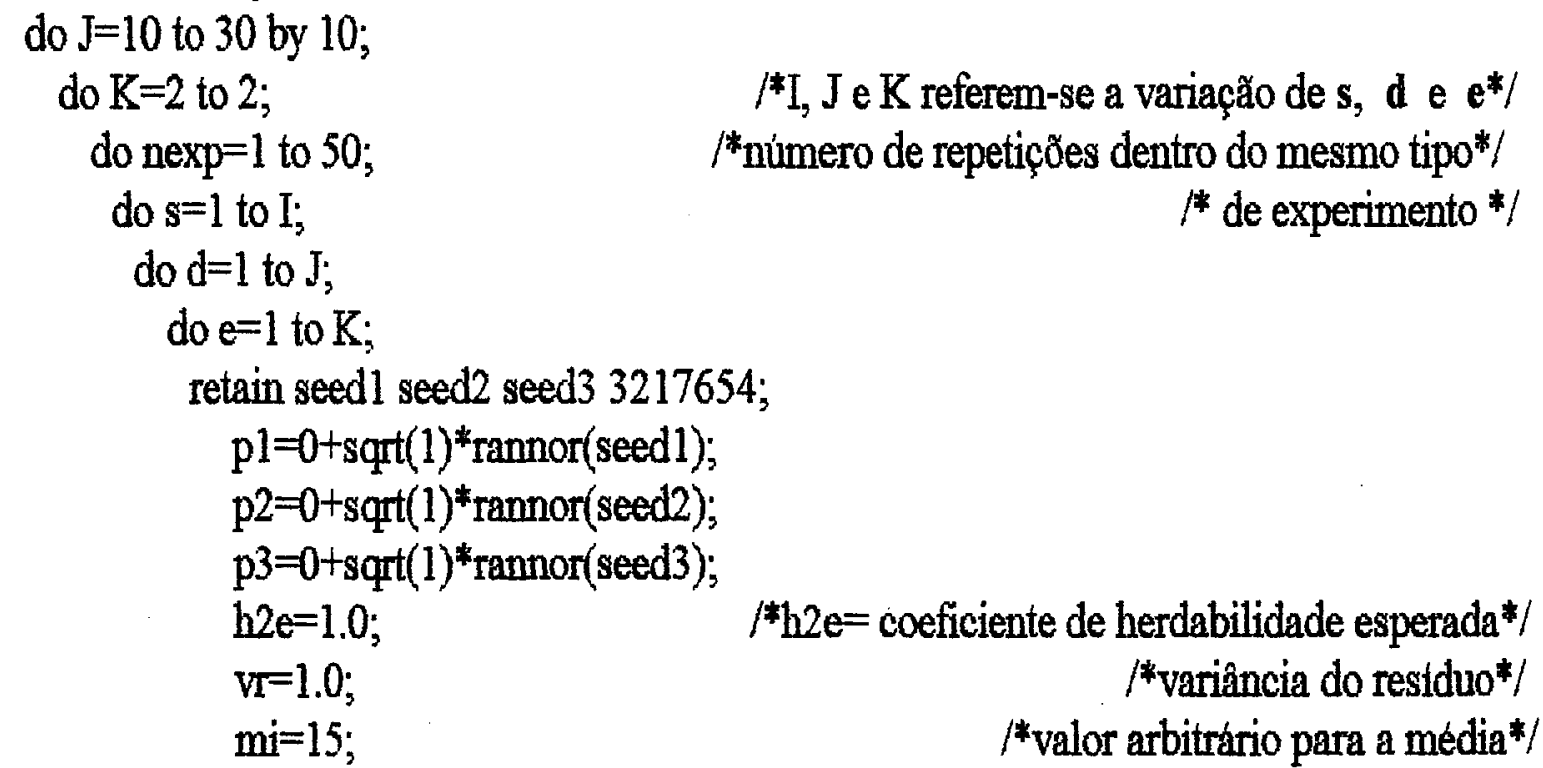

/*I, J e K referem-se a variação de s, d e e $\mathbf{e}^{* /}$ /*número de repetições dentro do mesmo tipo*/ * de experimento */ /*Sob a suposicão de valor nulo para a variância genética não-aditiva */ $\mathrm{vm}=\left(\left(\mathrm{h} 2 \mathrm{e}^{*} \mathrm{vr}\right) /\left(2^{*}(2-\mathrm{h} 2 \mathrm{e})\right)\right)$; vrep $=15+\mathrm{sqrt}(\mathrm{vm})^{*} \mathrm{p} 1+\mathrm{sqrt}(\mathrm{vm})^{*} \mathrm{p} 2+\mathrm{p} 3$;

if $\mathrm{I}<10$ and $\mathrm{J}<10$ then caso $=100^{*} \mathrm{I}+10^{*} \mathrm{~J}+\mathrm{K}$; else caso $=(10000 * \mathrm{I}+100 * \mathrm{~J}+10 * \mathrm{~K}) / 10$;

/*aso indica diferentes combinações de $s$, d e $e^{* /}$

$$
\text { output; }
$$

end;

end;

end;

end;

end;

end;

end;

keep s d e nexp caso vrep I J K; 
proc anova noprint outstat=saida?;

by caso nexp;

class $\mathrm{s} \mathrm{d}$;

model vrep $=\mathrm{s} \mathrm{d}(\mathrm{s}) / \mathrm{ss} 3 ; \quad$ /*realiza a análise de variância para cada experimento*

data saida3;

set saida2;

keep caso nexp_source_df ss;

data al;

/*guarda as SQ e Gl para s dentro de cada experiemnto*/

set saida3;

if_source ${ }^{\wedge}=' S$ then delete;

$\mathrm{gls}=\mathrm{df} ; \mathrm{sqs}=\mathrm{ss}$;

keep caso nexp gls sqs:

data a2;

/*guarda as SQ e GL para cada d(s) em cada experimento*/

set saida3;

if_source_ $\wedge=D(S)^{\prime}$ then delete;

$\mathrm{gld}=\mathrm{df} ; \mathrm{sqd}=\mathrm{ss} ;$

keep caso nexp gld sqd;

data a3;

/*guarda as SQ e GL para cada e[d(s)] em cada experimento*/

set saida3;

if_source_^=ERROR' then delete;

gle $=\mathrm{df} ;$ sqe $=\mathrm{ss}$;

keep caso nexp gle sqe;

data junta;

/*em cada linha há todas as informações de um experimento*

merge al a2 a3;

by caso nexp;

$\mathrm{I}=\mathrm{gls}+1 ; \mathrm{J}=(\mathrm{gld} / \mathrm{T})+\mathrm{l} ; \mathrm{K}=\mathrm{gle} /\left(\mathrm{I}^{*} \mathrm{~J}\right)+1$;

$\mathrm{Vl}=\mathrm{sqs} / \mathrm{gls}$;

$\mathrm{V} 2=$ sqd/gld;

$\mathrm{V} 3=$ sqe/gle;

$\mathrm{E}=\mathrm{v} 3$;

$\mathrm{D}=(\mathrm{v} 2-\mathrm{v} 3) / \mathrm{K}$ 
$\mathrm{S}=(\mathrm{vl}-\mathrm{v} 2) /(\mathrm{J} * \mathrm{~K})$

if $D<0$ then $D=0$;

if $S<0$ then $S=0$;

h2 $S=(4 * S) /(S+D+E)$

/Fórmula para o cálculo da estimativa do coef. herdabilidade*/

/*formulas necessárias para calcular a variância dos coef. de herdabilidade, só vale para dados balanceados*/

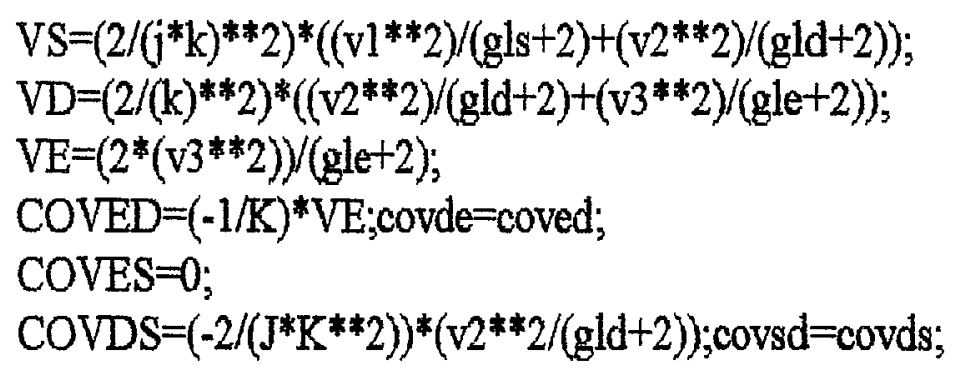

/*fómmula aproximada de DICKERSON (1969) para Variância do Coef. de Herdabilidade baseada na correlação entre meios-irmaos paternos*/

$\mathrm{Vh} 2 \mathrm{SD}=\left(16^{*} \mathrm{VS}\right) /\left((\mathrm{S}+\mathrm{D}+\mathrm{E})^{* *} 2\right) ;$

/*fórmula aproximada de OSBORNE \& PATERSON (1952) para a Variância do Coef. de Herdabilidade baseada na correlação entre meios-irmaos paternos*/

$\mathrm{Vh} 2 \mathrm{SOP}=(16 *((\mathrm{E}+\mathrm{D}) * * 2) * \mathrm{VS}+(\mathrm{S} * 2) *(\mathrm{VE}+\mathrm{VD}+2 * \mathrm{COVED}+2 * \mathrm{COVES})$

$\left.-2 * S^{*}(\mathrm{E}+\mathrm{D})^{*}(\mathrm{COVES}+\mathrm{COVDS})\right) /\left((\mathrm{S}+\mathrm{D}+\mathrm{E})^{* *} 4\right) ;$

/*formula de FALCONER (1960) para a Variância do Coef. de Herdabilidade baseada na correlação entre meios-irmãos paternos*/

$\mathrm{Vh} 2 \mathrm{SF}=(32 * \mathrm{~h} 2 \mathrm{~S}) /\left(\mathrm{I}^{*} \mathrm{~J} * \mathrm{~K}\right)$;

proc means $\operatorname{maxdec}=4$ mean $\min \max$;

title 'Var. da Herd. Estimada por Diferentes Metodos para $\mathrm{H} 2=1.0$ ';

var h2S Vh2Sf Vh2SD Vh2SOP;

by caso;

output out=estat 1 mean=média min=mínimo max-máximo; 
data estat2;

set junta;

keep caso h2S;

if $h 2 S^{\wedge}=0$ then $h 2 S=1 ;$ else $h 2 S=0$;

proc freq;

title Prob. de Obter Estimativas Negativas de Herdabilidade para $\mathrm{H} 2=1.0$;

tables h2S / nocum;

by caso;

run;

Observação: Este programa foi rodado para cada herdabilidade esperada, devido à falta de es paço em disco. 